\title{
Variability of massive stars with known spectral types in the Small Magellanic Cloud using 8 years of OGLE-III data ${ }^{\star}$
}

\author{
M. Kourniotis ${ }^{1,2}$, A. Z. Bonanos ${ }^{1}$, I. Soszyński ${ }^{3}$, R. Poleski ${ }^{3,4}$, G. Krikelis ${ }^{2}$, A. Udalski ${ }^{3}$, M. K. Szymański ${ }^{3}$, \\ M. Kubiak ${ }^{3}$, G. Pietrzyński ${ }^{3,5}$, Ł. Wyrzykowski ${ }^{3}$, K. Ulaczyk ${ }^{3}$, S. Kozłowski ${ }^{3}$, and P. Pietrukowicz ${ }^{3}$ \\ 1 IAASARS, National Observatory of Athens, 15236 Penteli, Greece \\ e-mail: [mkourniotis; bonanos] @astro.noa.gr \\ 2 Section of Astrophysics, Astronomy and Mechanics, Faculty of Physics, University of Athens, Panepistimiopolis, 15784 Zografos, \\ Athens, Greece \\ 3 Warsaw University Observatory, Al. Ujazdowskie 4, 00-478 Warszawa, Poland \\ ${ }^{4}$ Department of Astronomy, Ohio State University, 140 W. 18th Ave., Columbus OH 43210, USA \\ 5 Universidad de Concepción, Departamento de Astronomía, 160-C Casilla, Concepción, Chile
}

Received 16 October 2013 / Accepted 30 November 2013

\begin{abstract}
We present a variability study of 4646 massive stars in the Small Magellanic Cloud (SMC) with known spectral types from the catalog of Bonanos et al. (2010) using the light curves from the OGLE-III database. The goal is to exploit the time domain information available through OGLE-III to gain insight into the processes that govern the evolution of massive stars. This variability survey of massive stars with known spectral types is larger than any previous survey by a factor of 7 . We find that $60 \%$ of our sample (2766 stars) show no significant variability and 40\% (1880 stars) exhibit variability distributed as follows: 807 stars display low-amplitude stochastic variability with fluctuations in the $I$-band of up to $0.05 \mathrm{mag}, 443$ stars present irregular variability of higher amplitude (76\% of these are reported as variables for the first time), 205 are eclipsing binaries (including 101 newly discovered systems), 50 are candidate rotating variables, 126 are classical Cepheids, 188 stars exhibit short-term sinusoidal periodicity $(P<3$ days $)$ making them candidate "slowly pulsating B stars" and non-radial Be pulsators, and 61 periodic stars exhibit longer periods. We demonstrate the wealth of information provided in the time domain, by doubling the number of known massive eclipsing binary systems and identifying 189 new candidate early-type Be and 20 Oe stars in the SMC. In addition, we find that $\sim 80 \%$ of Be stars are photometrically variable in the OGLE-III time domain and provide evidence that short-term pulsating stars with additional photometric variability are rotating close to their break-up velocity.
\end{abstract}

Key words. binaries: eclipsing - galaxies: individual: SMC - stars: early-type - stars: variables: general - stars: massive stars: emission-line, Be

\section{Introduction}

The intrinsic variability of massive stars $\left(\gtrsim 8 M_{\odot}\right)$ is prominent from their main-sequence lifetime to their end-state evolution. Very massive stars exhibit strong photospheric winds as a result of radiation pressure (Kudritzki \& Puls 2000), yielding high mass-loss rates, which can cause line profile variability and possibly brightness variations (e.g., Richardson et al. 2011). Given the dependence of line driven winds and consequently mass loss rates on metallicity, a significant fraction of early-type stars close to the main sequence exhibit fast rotation in low-metallicity environments. These so-called "Be stars" eject mass that accumulates in a circumstellar disk, the establishment and dissipation of which results in spectroscopic and photometric variability. Other classes of early B-type stars, such as $\beta$ Cephei variables and slowly pulsating B stars (SPBs), display short-term periodic modulation in their light curves as a result of radial pulsations driven by the $\kappa$-mechanism, which is due to the iron-group

* Full Tables 1-10 are only available at the CDS via anonymous ftp to cdsarc.u-strasbg. fr $(130.79 .128 .5)$ or via http://cdsarc.u-strasbg.fr/viz-bin/qcat?J/A+A/562/A125 opacity bump. Low metal abundance appears to narrow or even make the instability strip of B-type pulsators vanish (Miglio et al. 2007). In particular, after adopting a chemical mixture representative of B stars in the SMC, no $\beta$ Cephei stars are expected at $Z<0.007$, while no SPBs are expected at $Z<0.005$ (Salmon et al. 2012).

On the other hand, the extrinsic variability of massive stars is mostly prominent in the case of eclipsing systems. The high binarity fraction of early-type OB stars (Sana et al. 2012; Chini et al. 2012) makes the detection of photometric and spectroscopic variability common. Eclipsing binaries provide a geometrical method for deriving accurate fundamental parameters of stars (Andersen 1991; Torres et al. 2010). Photometry combined with spectroscopy yields the physical radii of the systems, individual masses, and therefore, the surface gravities. Furthermore, effective temperatures and luminosities can be estimated, enabling an accurate and direct determination of the reddening and the distance to OB-type stars in galaxies of the Local Group (e.g., Bonanos et al. 2006, 2011). Measuring the fundamental parameters of massive stars accurately is of prime importance for constraining models of the formation and evolution of massive 
stars (e.g., Bonanos 2009). Eclipsing binary measurements also provide the most robust values for the upper mass limit (e.g., Bonanos et al. 2004; Rauw et al. 2004).

The main goals of this work are to identify new eclipsing binaries with massive components and to explore the Be phenomenon via photometric variability. Classical $\mathrm{Be}$ stars are B-type stars with luminosity classes III-V, which rotate close to their critical limit (Porter \& Rivinius 2003; Rivinius et al. 2013). They exhibit hydrogen Balmer emission, as well as emission of He I, Fe II, and sometimes Si II and Mg II, with double-peaked profiles, indicating the presence of a circumstellar, gaseous, quasi-Keplerian disk. Spectroscopy confirms the Be nature of a star; however, the transience of the phenomenon means that the method is not definitive. The modification of the disk owing to the fast rotation possibly combined with non-radial pulsations (Rivinius et al. 1998) is widely accepted as producing outbursts and fluctuations in brightness. Several explanations of the formation of the disk have been proposed, such as Bjorkman \& Cassinelli (1993), Cassinelli et al. (2002), and Rivinius et al. (2001). It has also been established that metallicity plays a major role in the frequency and the intensity of the Be phenomenon. Low metal abundance results in low mass-loss rates since the radiation pressure that is responsible for driving the stellar winds acts on the metal lines (Vink et al. 2001), therefore angular momentum loss remains low, and the star reaches a high $\Omega / \Omega_{\mathrm{c}}$ ratio, triggering the $\mathrm{Be}$ phenomenon. As a result, the fraction of earlyBe stars in the SMC is found to exceed the fraction in the Galaxy and the Large Magellanic Cloud (LMC; McSwain \& Gies 2005; Wisniewski \& Bjorkman 2006; Martayan et al. 2010), specifically in the second half of their main-sequence lifetimes. In addition, the duration and amplitude of the outbursts as ejections of matter from Be stars, appear longer and larger in the SMC than in the LMC (Sabogal et al. 2005).

Over the past two decades, the microlensing experiments MACHO (Alcock et al. 1993), the Optical Gravitational Lensing Experiment II (OGLE-II; Udalski et al. 1997), and EROS (Aubourg et al. 1995) have provided a database of light curves of a large number of massive stars located in the Magellanic Clouds and the Galaxy, motivating detailed studies of Be stars (Keller et al. 2002; Mennickent et al. 2002; de Wit et al. 2003; Sabogal et al. 2005, 2008) and other types of massive stars. Furthermore, surveys with the All Sky Automated Survey (ASAS; Pojmanski 2002) and the Hipparcos mission have motivated a couple of studies on variability as a function of spectral type. Szczygieł et al. (2010) exploited ten years of photometry provided by ASAS to probe for variability in $\sim 600$ massive stars in the LMC. They focused on bright, late spectral-type stars and found 117 variables of which $\sim 73 \%$ were red supergiants. Hubert \& Floquet (1998) used the four-year Hipparcos photometry of 289 Galactic Be stars with spectral types from O9.5 to A0, consisting of 110 epochs, to detect short-term, low-amplitude, periodic variability in $\sim 86 \%$ of early-Be stars. They also excluded the presence of short-lived outbursts in late-Be type galactic stars.

The goal of this paper is to take advantage of the eight-year, wide-field monitoring of the OGLE-III project, exploit the time domain and correlate variability with the spectral type of massive stars in the SMC. We used the catalog of 5324 massive stars with known spectral types compiled by Bonanos et al. (2010) from the literature. The paper is organized as follows. In Sect. 2, we give a brief description of the input spectral catalog and the OGLE-III survey that provided the light curve database. In addition, we describe the matching procedure and the constraints that resulted in the final list of stars studied. In Sect. 3, we present the method used to separate the "real" from the "spurious" variables and to search for periodic signals. In Sect. 4, we report our results on periodic and irregular variables. In Sect. 5, we discuss our results and derive statistics for stars exhibiting clear photometric variability. In Sect. 6, we summarize the main results of the present work.

\section{Input catalogs}

We used the catalog of 5324 massive stars in the SMC compiled by Bonanos et al. (2010), which contains their name, coordinates (J2000.0), spectral classification (typically accurate to one spectral sub-type and one luminosity class), and reference. The catalog consists of 12 Wolf Rayet, 277 O-type, 3069 early B-type, 602 late B-type, 1222 AFG-type, $107 \mathrm{~K}$ and M-type stars, 34 stars classified as "Be" (including $21 \mathrm{X}$-ray binaries) and treated in the present work as early B-type stars, and 1 supergiant $\mathrm{B}[\mathrm{e}]$ star.

Our goal is to match the stars from the catalog of Bonanos et al. (2010) to stars with light curves from the OGLE-III database. The OGLE-III survey covered 14 square degrees (41 fields; Udalski et al. 2008), which are centered on the densest regions of the SMC. The observational data were obtained between 2001 and 2009 with the 1.3 m Warsaw telescope at Las Campanas Observatory in Chile. The telescope was equipped with an eight-chip CCD mosaic camera with $8192 \times$ 8192 pixels and a field of view of about $35 \times 35$ arcmin (details on the instrumentation setup can be found in Udalski 2003). About $90 \%$ of the observations were collected in the Cousins $I$-band, while $10 \%$ were obtained in the Johnson $V$ photometric band.

\section{Matching procedure}

For each of the 5324 stars in the catalog, we examined all the OGLE-III light curves matched within a radius of $2^{\prime \prime}$, given the $1^{\prime \prime}$ accuracy in the coordinates of the stars of the input cata$\log$. A total of 582 stars had no match since they correspond to stars not covered by the OGLE-III fields or to very bright stars that are saturated in the OGLE-III images, including all $108 \mathrm{~K}$ and $M$ supergiants of the catalog. We found that 2188 stars only have one match. For 2554 stars, more than one light curve was detected within $2^{\prime \prime}$. In these cases, we selected the brightest source as the correct match unless there was a source closer than $1^{\prime \prime}$ that was within 0.5 mag of the brightest match, so as to avoid selecting faint instrumental artifacts that usually lie in the vicinity of bright stars.

In total, 485 close matches were selected using this criterion. To confirm our selection, we compared the mean $I$ and $V$ values of the candidate light curves to the respective ones from the Magellanic Clouds Photometric Survey (MCPS; Zaritsky et al. 2002), as provided in Bonanos et al. (2010) when available. We set a differential limit of $0.5 \mathrm{mag}$, below which the selected light curve was considered to be the correct match, in order not to reject counterparts of variable stars with outbursts or deep eclipses. A total of 70 stars were found to exceed the particular limit in at least one band. We kept five that proved to be eclipsing binaries, 4 Cepheids and 14 stars with significant irregular variability, as we discuss later. The remaining 47 were excluded from our study, since they presented constant light curves or even low-amplitude variability across the OGLE-III monitoring period, which could not justify the discrepancy of 0.5 mag or 


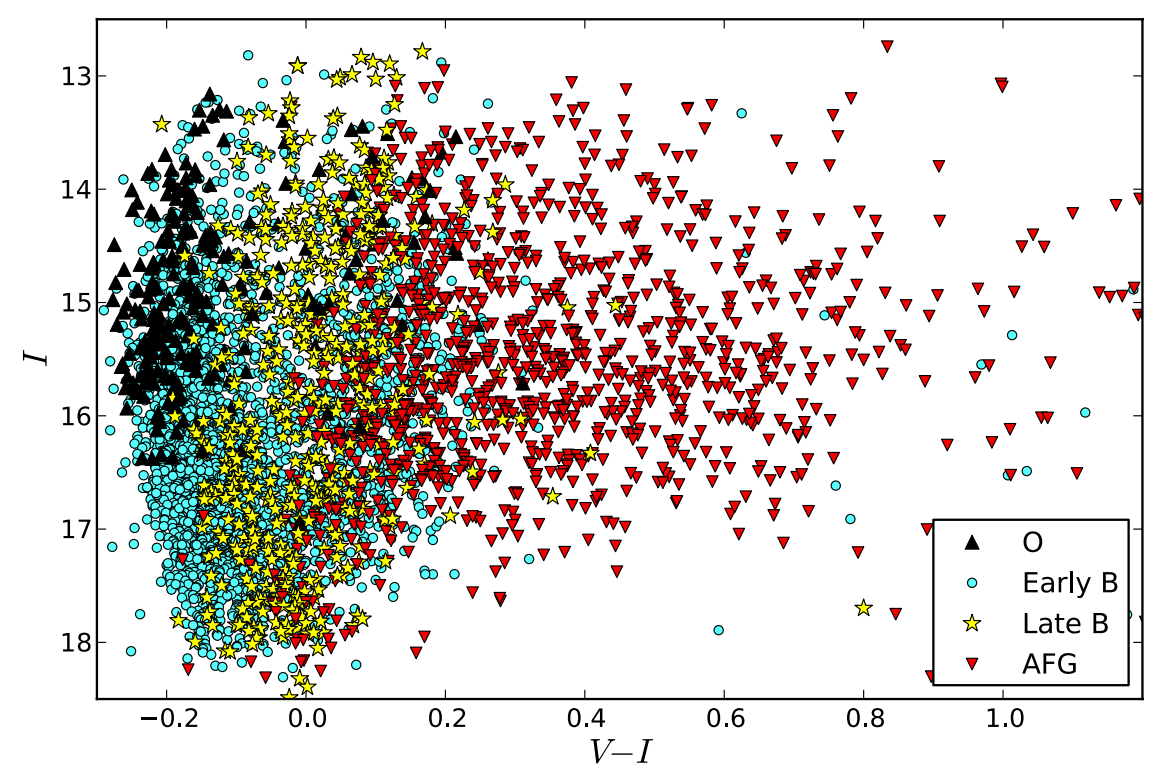

Fig. 1. $I$ vs. $V-I$ color-magnitude diagram for the 4614 stars from our sample of 4646 stars that have both $I$ and $V$-band OGLE-III photometry. The spectral types are labeled as follows: O stars as black triangles, early-B stars as cyan circles, late-B stars as blue stars, and AFG-type stars as red inverted triangles. Only $1.3 \%$ of early-B type stars exhibit very red colors due to contamination.

more. However, we caution that the photometry of MCPS was conducted before the OGLE-III survey, and therefore, some of these rejected stars might have truly exhibited variable behavior in the past.

We ended up with a catalog of 4646 stars with I-band magnitudes between 12.6-19.4, having also excluded 15 lightcurves with fewer than 100 epochs, because the median number of epochs for the matched light curves in the $I$-band is 698, as well as 31 lightcurves that correspond to bright stars close to saturation or to known types of artifacts in the OGLE photometry. We also excluded three stars verified as double entries ${ }^{1}$ in the input catalog. The color-magnitude diagram of the catalog is presented in Fig. 1. It consists of 4614 stars, since 32 stars were not measured in the $V$-band. We note that the very reddened colors $(V-I>0.5 \mathrm{mag})$ of some early and late B-type stars are due to contamination by hot spots in the interstellar medium, as recently shown by Sheets et al. (2013) and Adams et al. (2013). The percentage of early B-type stars affected by contamination is only $1.3 \%$.

\section{Analysis}

Our analysis of the 4646 OGLE-III light curves is based on the four statistical moments of the $I$-band light curve: the mean magnitude, standard deviation, skewness, and the kurtosis. We used a $2 \sigma$ cut over the mean to remove possible outliers from each light curve and recalculated the statistics. To distinguish between real variables and non-variables, we used the $\sigma_{I}$ vs. $I$ plane where $\sigma_{I}$ is the standard deviation of the $I$-band light curve points (Fig. 2). In this plane, the real variables are defined according to Graczyk \& Eyer (2010) for the OGLE-II database of light curves in the LMC as those that lie above their proposed, empirical curve. Our goal is to search for significant variability so we decided to add a shifted, elevated curve and classified 935 stars that lie above as high-amplitude variables, 945 stars that were found between the two curves as lowamplitude variables, and 2766 stars that lie below the primary

\footnotetext{
1 NGC346-016/2dFS5100, NGC330-110/SMC5_037341 and OGLEtd9175323/Hodge53h53-47.
}

curve as constant. In fact, since the primary curve is empirical, we expect partial overlap between the low-amplitude region and that of the non-variables. Our low-amplitude variables present variability $\Delta I \lesssim 0.05 \mathrm{mag}$.

For the detection of eclipsing binaries (EBs), we followed the method introduced by Graczyk \& Eyer (2010) based on the skewness and the kurtosis of the distribution of magnitudes. We initially focused on those stars in the region of the skewnesskurtosis diagram where we expect eclipsing binaries to lie. Cepheids tend to be more symmetric with negative kurtosis, and as a result they are found in the lower region of this diagram. Figure 3 shows the location of the Cepheids from our catalog and both our newly discovered and the known eclipsing binaries on the skewness-kurtosis diagram. The light curves were searched for periodicity using the Analysis of Variance (AoV) algorithm (Schwarzenberg-Czerny 1989) in the frequency range of 0.0001-7 cycles per day and were folded to the period corresponding to the frequency with the highest peak in the power spectrum. The discovery of numerous short- and long-term sinusoidally varying periodic stars located close to the $(0,0)$ point of the skewness-kurtosis diagram motivated us to extend our periodic search. Eventually, all low-amplitude variables, as well as some of the high-amplitude variables, which were strongly suspected of being periodic after visual inspection of their raw light curves, were inspected with the period search algorithm. This yielded a significant number of short- and long-period variables representative of pulsating and rotating variables, as discussed in Sect. 4. In addition, we identified 13 eclipsing binaries that were found to lie outside the borders of their expected region since they were either very detached with few downward outliers or had rather symmetric light curves.

\section{Results}

\subsection{Periodic variables}

\subsubsection{Eclipsing binaries}

The discovery of new eclipsing binaries with high-mass components is the first step toward populating the high-mass end of 


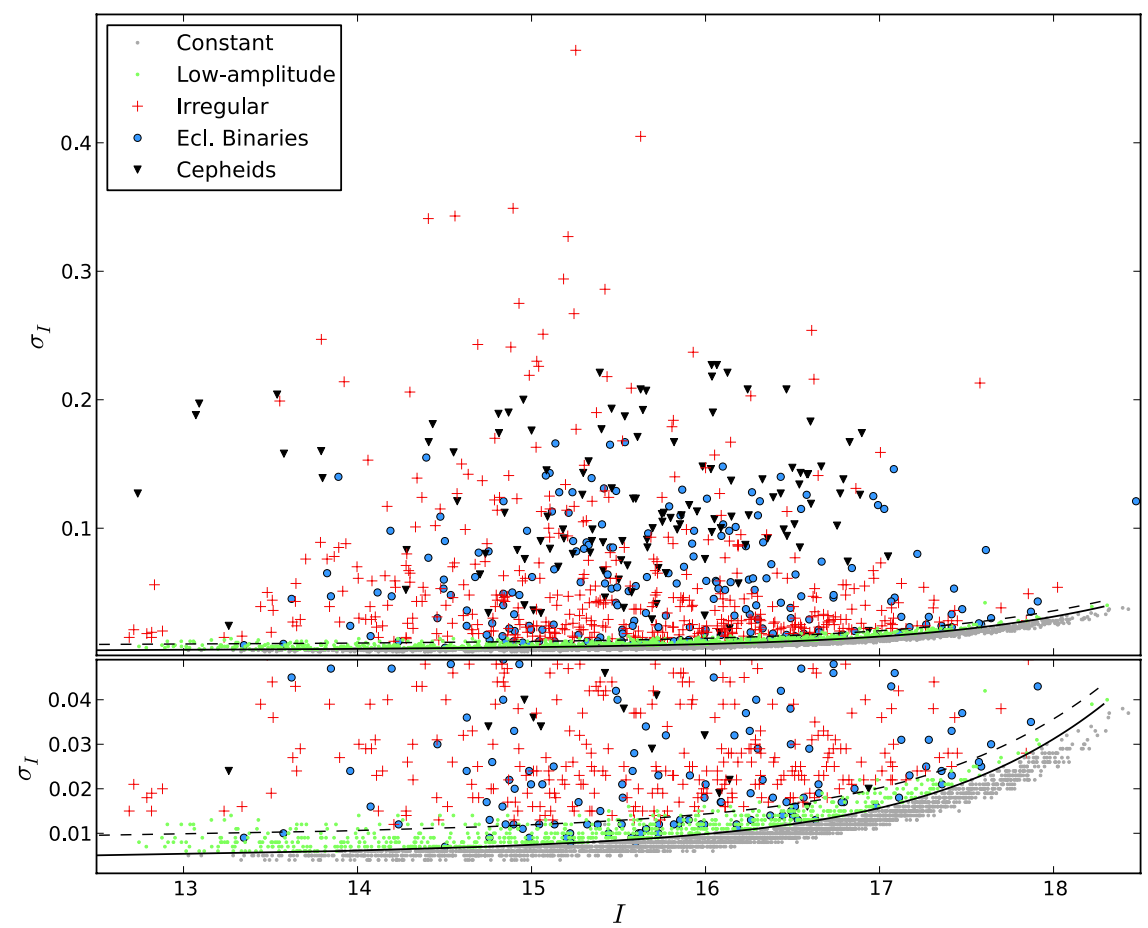

Fig. 2. $\sigma_{I}$ vs. $I$ diagram for the 4646 stars studied in this work. The solid curve is used to separate real from spurious variables (as proposed by Graczyk \& Eyer 2010), while the region between the solid and the dashed curves defines the low-amplitude variables. A zoomed-in region is presented in the lower panel. The irregular variables (crosses), the Cepheids (inverted triangles), and most of the EBs (open circles) lie above the dashed curve.

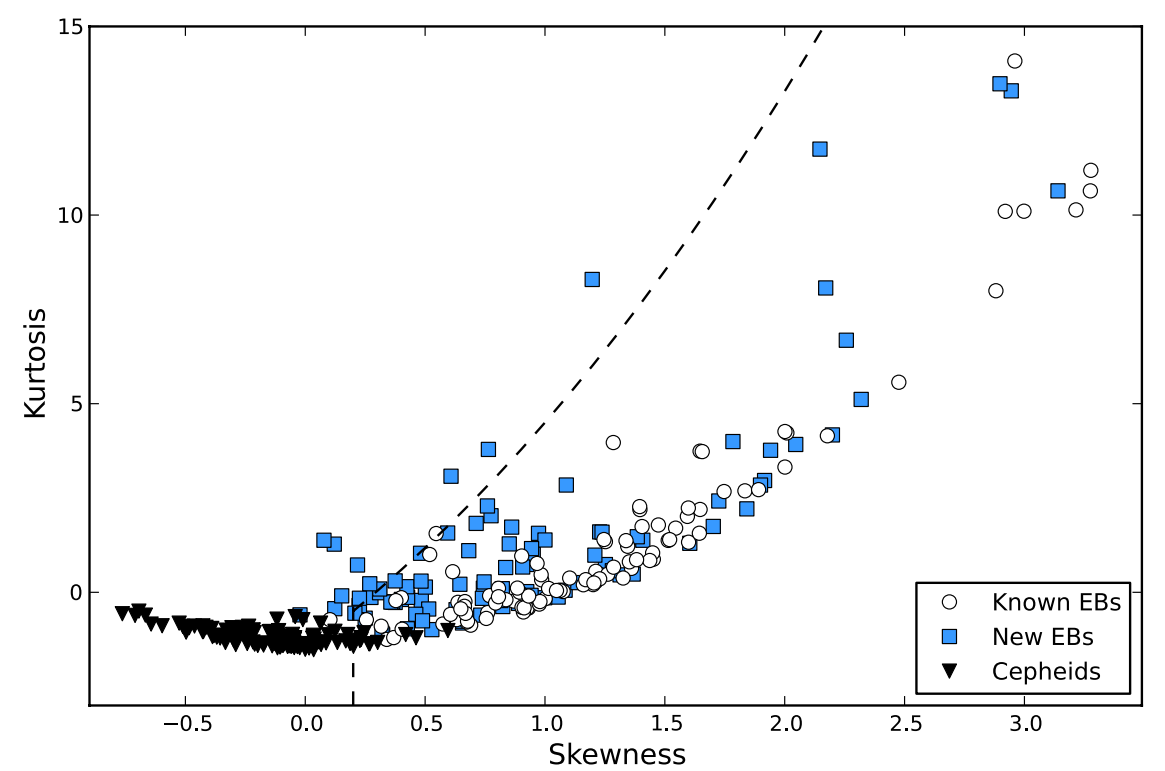

Fig. 3. Skewness-Kurtosis diagram for the eclipsing binaries and the Cepheids identified in the spectral catalog. New eclipsing binaries are indicated with blue squares, and known eclipsing binaries with circles. Eclipsing binaries are expected to be found below the dashed curve for skewness values higher than 0.2, as defined by Graczyk \& Eyer (2010). However, the method was found to miss systems with sparsely sampled eclipses (above the dashed curve) or with rather symmetric light curves (close to $(0,0)$ ).

the mass-radius relation with accurate measurements derived dynamically using double-lined spectroscopic eclipsing binaries. During the periodic search described in the previous section, we identified 205 eclipsing binaries. A total of 20 of them present a displacement of the secondary eclipse with time, which is an indication of apsidal motion. In addition, the EB 2dFS2780 $(P=5.602226$ days $)$ was found to exhibit additional longterm periodicity $(P 2=148.80952$ days $)$ and is a candidate double-periodic variable. This class of variables was first introduced in the SMC by Mennickent et al. (2003) and has also been investigated in the LMC (Mennickent et al. 2003; Poleski et al. 2010). Their enigmatic nature reveals a short-term period due to the orbital motion of a semi-detached binary and a long-term period that lasts $\sim 33$ times the orbital period. The long-term period probably stems from the modulation of a circumbinary disk (Mennickent et al. 2008). We also identified two 
transient EBs 2dFS2090 and 2dFS3560 caused by orbital precession. The former is an EB with a period of 1.420025 days that only presents an additional EB observed in the middle of the OGLE-III time domain. The latter is a transient EB with a period of 6.223004 days, which appears to vanish close to the end of the monitoring period.

We searched each EB individually in the VizieR catalog, using a 3 " search radius, and found 101 systems without any previous reference to their eclipsing nature. Of these 101 systems, 8 are O-type binaries, 85 early B-type, and 7 late B-type, and only one has an F-type. In addition, nine of the new EBs have already been classified as spectroscopic binaries by Evans et al. (2006) and Hunter et al. (in prep.). Table 1 presents the main characteristics of the 101 new EBs sorted by spectral type, listing the star name(s), spectral type, coordinates in degrees (J2000.0), values of the skewness and kurtosis, the OGLE-III database number, mean magnitudes in the $I$ and $V$ bands, and period (days). We flag whether it is a reported single, double, or undefined spectroscopic binary, and then note the systems with apsidal motion, the transient EBs, and the double-periodic variable. Representative phased light curves of six newly discovered eclipsing binaries are presented in Fig. 4, along with their names, coordinates, spectral types, and derived periods. Phase zero corresponds to the deeper eclipse.

The OGLE-III observations provide us with high-quality, long-term light curves for the 104 known EBs, so that our derived periods in many cases are more precise than those reported in the literature. Figure 5 presents OGLE-III light curves of six previously known eclipsing binaries. Table 2 presents the 104 known eclipsing binaries as in Table 1, along with the most recent reference to the work that reports the eclipsing nature. The input catalog contains 45 EBs that have already been analyzed by Hilditch et al. (2005) and Harries et al. (2003). We have also identified 59 EBs, using the VizieR catalog, which have been reported in previous works, mainly by Wyrzykowski et al. (2004) and Faccioli et al. (2007). Six of these have been spectroscopically confirmed. We also note the case of star 2dFS1636, which was reported to have composite features of A and F type stars (Evans et al. 2004). We found it to be an eclipsing binary, which suggests binarity as a natural explanation for stars exhibiting such composite features in their spectra. This star was reported by Faccioli et al. (2007) as an eclipsing binary, however we are the first to make the connection to its spectral type.

\subsubsection{Candidate rotating variables/Overcontact binaries}

Rotating variables owe their variations in brightness to nonuniform surface features or to the non-spherical shape of distorted components within binary systems. The most common classes of rotating variables include the chemically peculiar stars (CPs) and ellipsoidal variables. CPs are stars in the upper part of the main-sequence with B or A spectral types, which present an inhomogeneous surface distribution of chemical elements and yield near-sinusoidal light curves as a result of stellar rotation. Variability is often attributed to strong and stable magnetic fields (e.g., Lüftinger et al. 2010; Krtička et al. 2012), although cases with weak magnetic fields have been reported as well (e.g., Krtička et al. 2009; Shulyak et al. 2010). The amplitude of the light variations decreases with increasing wavelength (Catalano et al. 1991). Ellipsoidal variables are close binaries with non-spherical components due to tidal interactions, whose folded light curves present sinusoidal morphology (Soszynski et al. 2004). In some cases, overcontact eclipsing binaries can

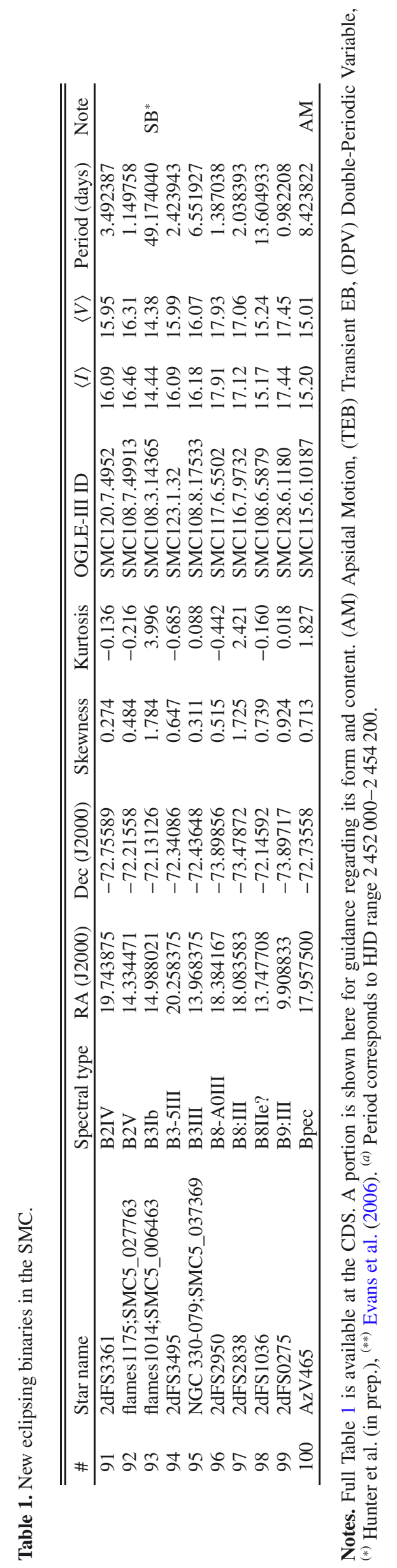



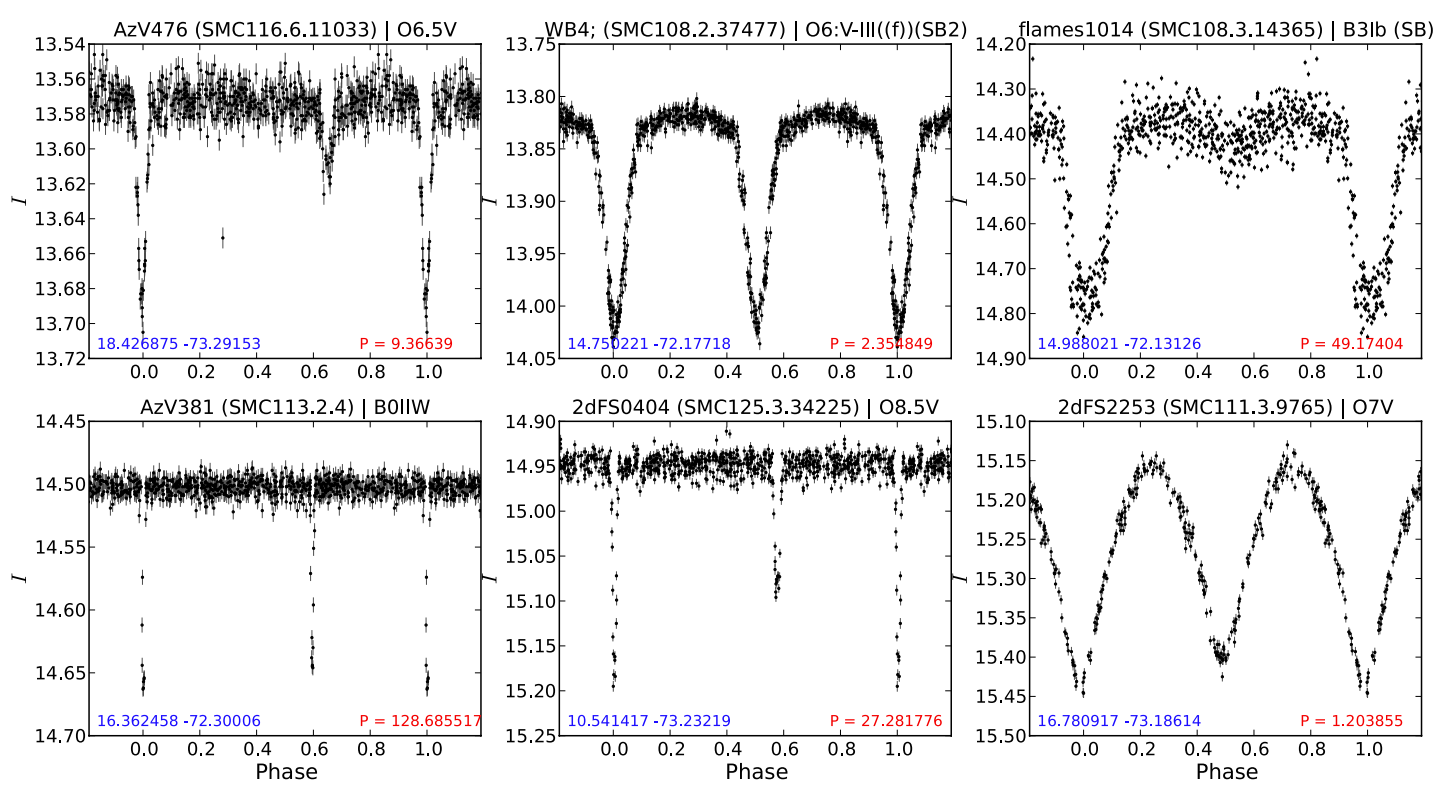

Fig. 4. Phased light curves of 6 newly discovered eclipsing binaries, along with their names, OGLE-III field and database numbers, spectral types, coordinates (J2000.0), and derived periods (in days), demonstrating the range of spectral types, luminosity classes, and binary configurations included in our sample. Error bars are indicated on each point.
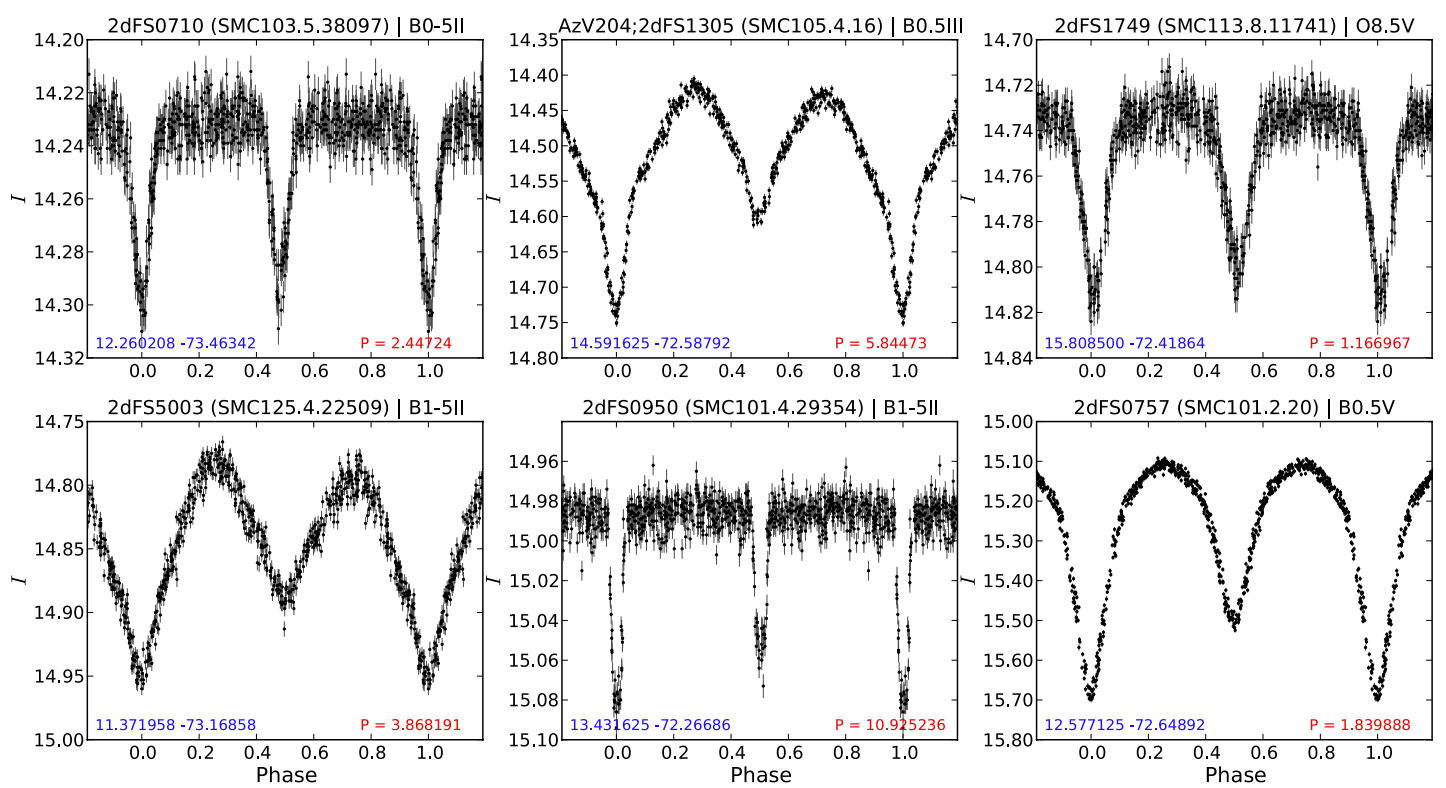

Fig. 5. Phased light curves of 6 known eclipsing binaries, as in Fig. 4. For many of these systems, their spectral types are presented for the first time.

produce ellipsoidal-like light curves (although of higher amplitude; Prša et al. 2011) especially if their light curves are noisy. Since these categories of variables produce identical light curves that can also be misclassified as pulsating stars, we decided to flag a star as a "candidate rotating variable" if it presents a nearsinusoidal light curve with unequal minima/maxima, since this is an indication of tidal distortion effects for an ellipsoidal star or uneven surface distribution of elements. We also include stars with equal minima that have been spectroscopically identified as binaries. A periodic variable that does not meet the requirements of an eclipsing binary or a candidate rotating variable has been flagged as a short- or a long-periodic variable, as discussed in Sect. 4.1.4. In Table 3 we list the parameters of the 50 candidate rotating variables or overcontact binaries, and we report the cases where the star has been classified in previous works as an eclipsing binary. Figure 6 presents the OGLE-III phased light curves of six candidate rotating variables.

\subsubsection{Cepheids}

The Cepheids in the SMC have already been the subject of extended study by Soszyński et al. (2010) using photometry provided by OGLE-III. However, the spectroscopic study of Cepheids has been more limited, even though it is crucial for understanding the properties of the stars themselves, their use as distance indicators (e.g., Romaniello et al. 2008), and as tracers of abundance gradients in galaxies (e.g., Andrievsky et al. 2002). We identified 126 classical Cepheids among the stars in 
M. Kourniotis et al.: Variability of massive stars in the SMC

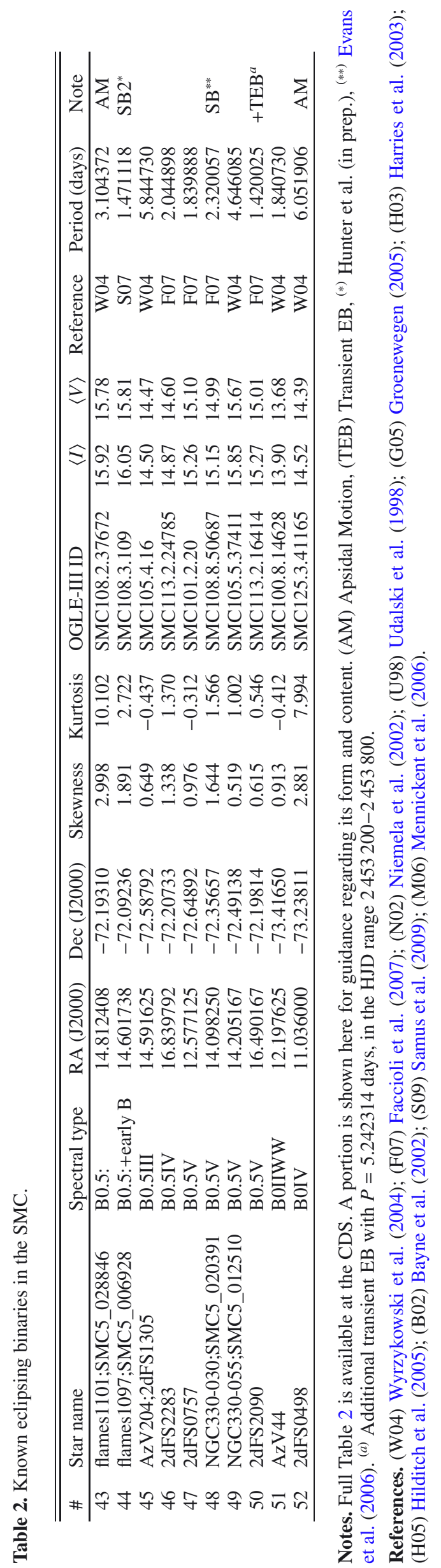

our catalog. They have already been reported in Soszyński et al. (2010) and we provide spectral types for these, finding 33 A-type stars, 85 F-type stars, 3 G-type stars, and 5 stars with composite features of both A and F stars (Evans et al. 2004), which are likely non-eclipsing binaries. Table 4 lists the main characteristics of the Cepheids, as in Table 1. Figure 7 presents the period-luminosity (PL) diagram for the matched Cepheids, in the reddening-independent Wesenheit index $W_{I}=I-1.55(V-I)$. We find that A-type Cepheids have $P<5.3$ days, while F-type Cepheids span the whole range of periods as expected, when considering the shape of the instability strip on the HertzsprungRussell (HR) diagram, which confines the A-type luminosity range to lower mass stars, while F-type stars span a wider luminosity range.

\subsubsection{Other periodic stars}

The remaining 249 periodic stars that we detected present mostly sinusoidal behavior and correspond to pulsating stars, rotating variables with starspot modulations, or ellipsoidal variables with equal minima. We classified these stars as "short-" and "longperiod" variables, when presenting periods shorter and longer than three days. Moreover, we flagged variables as "periodic with extra variability" when their periodic signal varies in amplitude or is modulated with a baseline variability of $\Delta I \gtrsim$ 0.05 mag. In Tables 5 and 6 , we present the main characteristics of the 138 short-periodic and 50 short-periodic stars with extra variability, respectively. Similarly, Tables 7 and 8 list the characteristics of the 45 long-periodic and 16 long-periodic with extra variability. Additionally, we note whether the star has been reported as an eclipsing binary in the literature. We have also matched eight long-period variables with the "Type-3" stars defined by Mennickent et al. (2002). By subtracting the longperiodic modulation, we found four $\operatorname{stars}^{2}$ that exhibit short-term periods that are equivalent to the expected orbital periods of double-periodic variables, as discussed in Sect. 4.1.1. In Fig. 8 we show four representative light curves of variables with short and long periodicity, one from each category described above.

\subsection{Irregular variables}

The morphology of the light curves of the high-amplitude variables, as defined from the $\sigma_{I}$ vs. $I$ plane, can be described as a combination of irregular, short-, or long-term outbursts with amplitudes up to $\Delta I \sim 1 \mathrm{mag}$ and/or trends with time scales of hundreds to thousands of days. Based on the classification presented in Keller et al. (2002) for blue variables in the LMC, we (re)defined four modes of variability for the $I$-band as follows:

- Bumper events - increase in magnitude from the baseline value up to $\Delta I \sim 1$ mag within a time range of 100-1500 days.

- Flicker events - short-term outbursts with an amplitude $\Delta I \gtrsim$ $0.05 \mathrm{mag}$, usually followed by exponential decay, and a duration of tens of days or even longer for larger amplitude outbursts.

- Monotonic events - long-term trend across the OGLE-III time domain, with an amplitude $\Delta I \gtrsim 0.05 \mathrm{mag}$.

- Fading events - decrease in magnitude up to $\Delta I \sim 0.6$ mag and duration up to 1000 days.

The Be phenomenon is known to be enhanced at low metallicities causing outbursts to last longer and have larger amplitudes

2 2dFS1126, 2dFS0309, 2dFS0435, 2dFS0096.

A125, page 7 of 19 
Table 3. Rotating variables/Overcontact binaries.

\begin{tabular}{lllcclrrrr}
\hline \hline$\#$ & Star name & Spectral type & RA (J2000) & Dec (J2000) & OGLE-III ID & $\langle I\rangle$ & $\langle V\rangle$ & Period (days) & Note \\
\hline 1 & AzV387;2dFS2050 & O9.5III & 16.416458 & -72.26822 & SMC113.2.8 & 14.11 & 13.87 & 2.972105 \\
2 & 2dFS2698 & B0-5II & 17.676917 & -73.32697 & SMC116.6.129 & 15.85 & 15.71 & 6.405136 \\
3 & 2dFS0300 & B0-5IV & 10.037958 & -73.64939 & SMC128.5.15134 & 16.63 & 16.44 & 2.368890 & W04 \\
4 & 2dFS2455 & B0-5IV & 17.176375 & -72.32797 & SMC113.1.31538 & 16.02 & 15.94 & 3.528916 & W04 \\
5 & 2dFS0359 & B0-5IV & 10.308958 & -73.42589 & SMC125.7.14593 & 16.55 & 16.69 & 1.667506 & \\
6 & 2dFS0010 & B0-5V & 7.349375 & -73.73997 & SMC138.4.5904 & 17.32 & 17.30 & 3.561880 & 1.320202 \\
7 & 2dFS1421 & B0-5V & 14.956333 & -72.54781 & SMC105.4.5344 & 16.49 & 16.40 & 4.326558 \\
8 & 2dFS2295 & B0-5V & 16.875167 & -73.43469 & SMC111.2.2145 & 16.56 & 16.51 & 0.856288 \\
9 & 2dFS2959 & B0-5V & 18.426875 & -72.63375 & SMC115.3.162 & 16.86 & 16.67 & 1.062355 \\
10 & 2dFS3304 & B0-5V & 19.517333 & -72.65019 & SMC120.6.146 & 16.80 & 16.68 & \\
\hline
\end{tabular}

Notes. Full Table 3 is available at the CDS. A portion is shown here for guidance regarding its form and content. ${ }^{(*)}$ Hunter et al. (in prep.), ${ }^{(* *)}$ Evans et al. (2006), ${ }^{(* *)}$ Martayan et al. (2007).

References. (W04) Wyrzykowski et al. (2004); (D08) Diago et al. (2008); (U98) Udalski et al. (1998); (M10) Mennickent et al. (2010).

Table 4. Known Cepheids in the SMC.

\begin{tabular}{rllllrrlrrr}
\hline \hline$\#$ & Star name & Spectral type & RA (J2000) & Dec (J2000) & Skewness & Kurtosis & OGLE-III ID & $\langle I\rangle$ & $\langle V\rangle$ & Period (days) \\
\hline 1 & 2dFS1914 & A0II & 16.165833 & -72.31958 & -0.227 & -1.049 & SMC113.8.39669 & 15.45 & 16.12 & 3.35206 \\
2 & 2dFS1472 & A2Iab & 15.115958 & -73.37806 & -0.271 & -0.976 & SMC106.2.30024 & 16.38 & 17.10 & 2.18004 \\
3 & 2dFS1719 & A2Ib & 15.737167 & -73.41747 & 0.087 & -1.341 & SMC111.7.58 & 15.51 & 16.26 & 5.19790 \\
4 & 2dFS1269 & A3Ib & 14.467083 & -74.10453 & -0.103 & -1.429 & SMC107.8.8007 & 16.75 & 17.17 & 1.08680 \\
5 & 2dFS2327 & A3Ib & 16.936458 & -73.19008 & -0.048 & -1.400 & SMC111.3.12630 & 16.22 & 16.74 & 1.54276 \\
6 & 2dFS2460 & A3Ib & 17.185833 & -72.69247 & -0.316 & -1.027 & SMC110.3.10311 & 15.63 & 16.45 & 3.71093 \\
7 & 2dFS0667 & A3II & 11.973833 & -72.83844 & 0.124 & -1.033 & SMC100.5.37708 & 15.98 & 16.41 & 3.28218 \\
8 & 2dFS0960 & A3II & 13.460917 & -74.02661 & -0.526 & -0.817 & SMC103.1.7083 & 16.11 & 16.80 & 2.70204 \\
9 & 2dFS1403 & A3II & 14.898833 & -73.05503 & -0.428 & -1.044 & SMC106.4.28330 & 17.04 & 17.66 & 0.68047 \\
10 & 2dFS2353 & A3II & 16.990958 & -73.30878 & -0.462 & -0.895 & SMC111.3.3080 & 16.50 & 17.02 & 1.85038 \\
\hline
\end{tabular}

Notes. Full Table 4 is available at the CDS. A portion is shown here for guidance regarding its form and content.
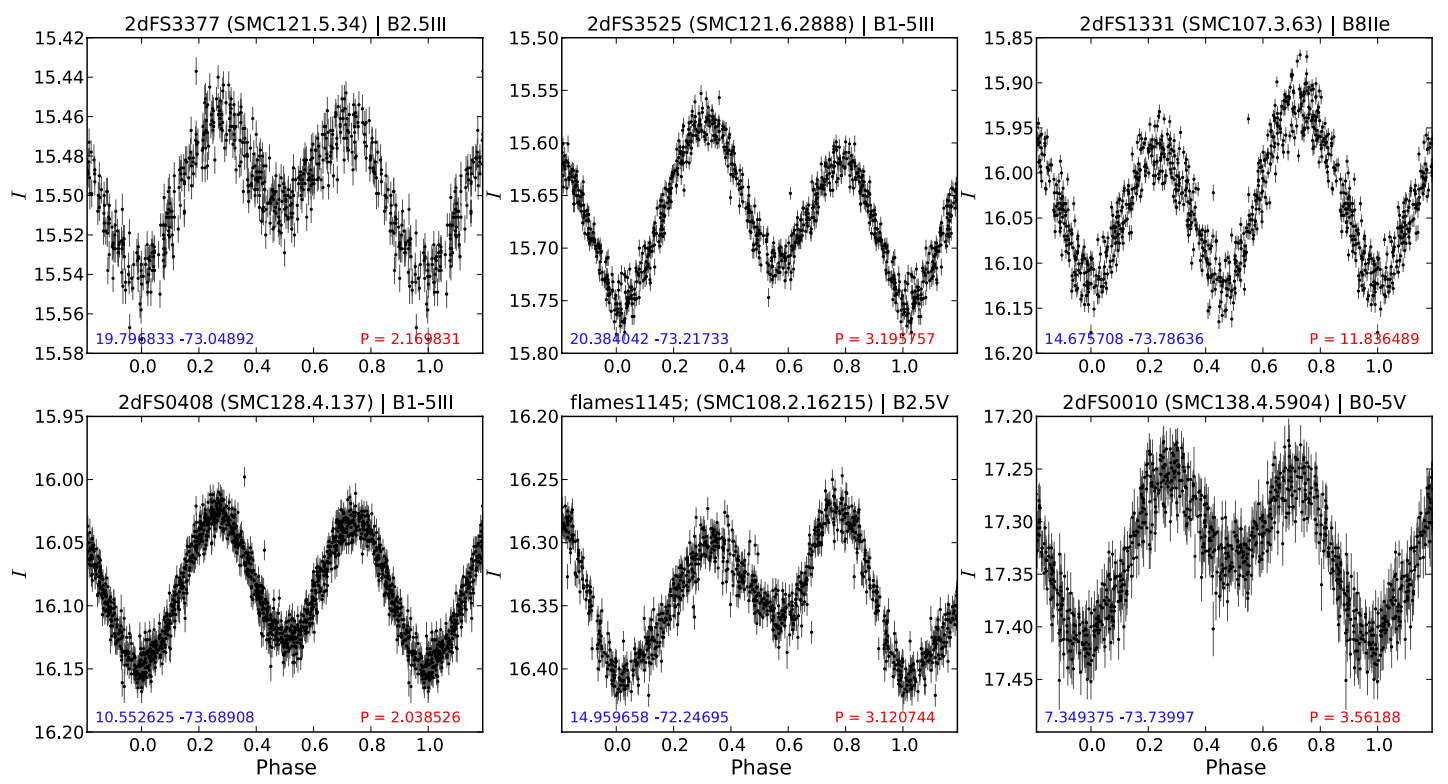

Fig. 6. Phased light curves of 6 candidate rotating variables, as in Fig. 4. Sinusoidally periodic variables with unequal minima are included in this category.

than in the LMC (Sabogal et al. 2005). The bumpers and the fading events reported in the LMC last up to 800 and 600 days, respectively. Variables with this behavior in our sample are found to have outbursts exceeding 1000 days in some cases. In addition, the $V$-band amplitudes exceed - although not significantly those reported in the LMC for the same type of photometric variability. Figure 9 presents eight stars with typical bumper, flicker, monotonic, and fading events. Bumper and flicker modes are equivalent to the type-1 hump-like and sharp outbursts, respectively, as defined by Mennickent et al. (2002). In total, 443 stars were flagged as "irregular" and are listed in Table 9. In the last column we note light curves dominated by a single mode. We cross-matched our irregular variables in the VizieR catalog and also against the photometric study in the SMC by 
M. Kourniotis et al.: Variability of massive stars in the SMC

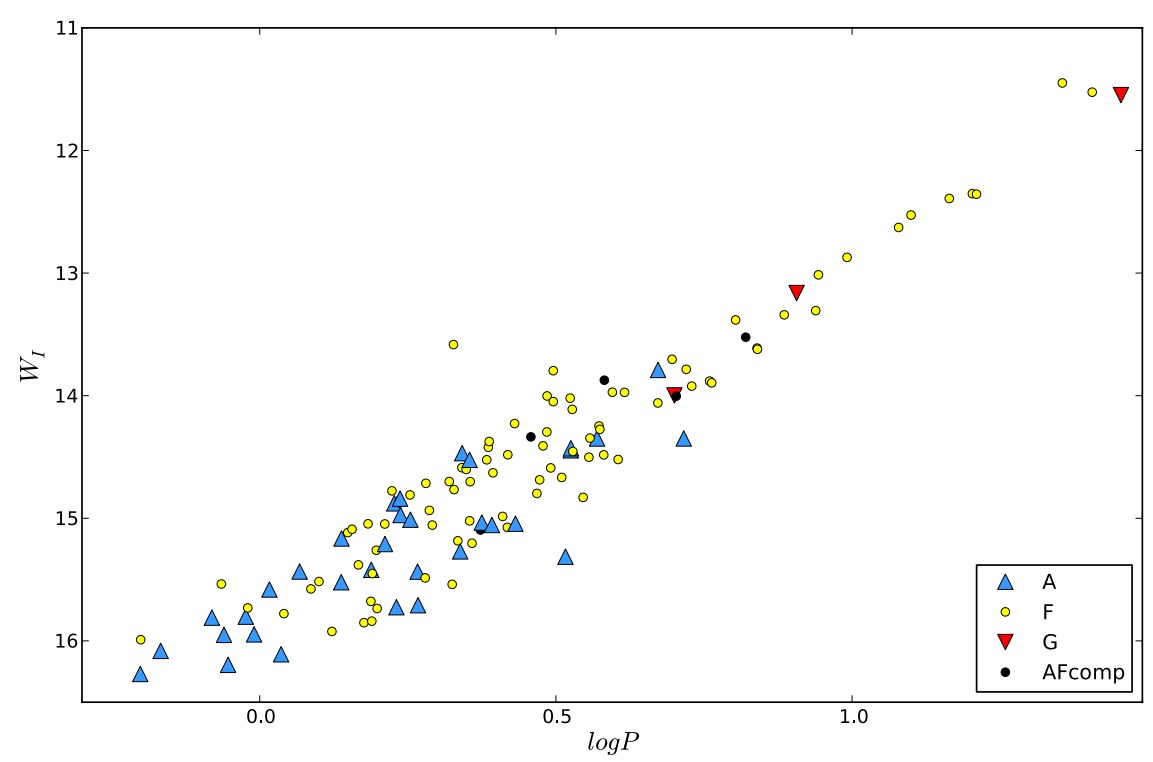

Fig. 7. Period-luminosity diagram for the Cepheids in the SMC that are included among our variables. Green triangles represent A-type stars, yellow circles F-type, red inverted triangles G-type, and black circles stars with composite features of A and F-type (AFcomp), which we propose to be binary systems.

Table 5. Short-period stars in the SMC.

\begin{tabular}{lllrllrrrr}
\hline \hline$\#$ & Star name & Spectral type & RA (J2000) & Dec (J2000) & OGLE-III ID & $\langle I\rangle$ & $\langle V\rangle$ & Period (days) & Note \\
\hline 22 & 2dFS1910 & B0-5IV & 16.156542 & -72.19458 & SMC113.7.40193 & 16.48 & 16.32 & 0.705250 & \\
23 & 2dFS2522 & B0-5IV & 17.290375 & -73.45019 & SMC111.2.4284 & 16.77 & 16.60 & 0.993426 & \\
24 & 2dFS3214 & B0-5IV & 19.187333 & -73.30769 & SMC116.3.7028 & 16.40 & 16.47 & 0.602205 & \\
25 & 2dFS3518 & B0-5IV & 20.370875 & -72.75442 & SMC120.2.45 & 15.97 & 15.90 & 0.579404 & W04 \\
26 & 2dFS1551 & B0-5IVe & 15.320458 & -72.76842 & SMC105.2.37523 & 16.44 & 17.74 & 0.534116 & \\
27 & 2dFS1142 & B0-5IVe? & 14.085417 & -73.60042 & SMC106.8.10982 & 16.31 & 16.39 & 1.241582 & \\
28 & 2dFS1773 & B0-5IVe? & 15.874375 & -72.97458 & SMC110.8.4493 & 16.75 & 16.85 & 0.309992 & \\
29 & 2dFS2164 & B0-5IVe? & 16.612167 & -72.28303 & SMC113.2.4478 & 16.16 & 16.24 & 0.656981 & \\
30 & 2dFS0205 & B0-5V & 9.520958 & -73.54000 & SMC125.8.16896 & 17.05 & 16.88 & 0.381540 & BCEP(+0.275963d) \\
31 & 2dFS0421 & B0-5V & 10.619042 & -72.65756 & SMC126.3.431 & 17.58 & 17.47 & 0.612584 & \\
\hline
\end{tabular}

Notes. Full Table 5 is available at the CDS. A portion is shown here for guidance regarding its form and content. (BCEP): Candidate $\beta$ Cephei.

References. (W04) Wyrzykowski et al. (2004); (D08) Diago et al. (2008); (I04) Ita et al. (2004).

Table 6. Short-period stars with extra variability in the SMC.

\begin{tabular}{|c|c|c|c|c|c|c|c|c|c|}
\hline \# & Star name & Spectral type & RA (J2000) & Dec (J2000) & OGLE-III ID & $\langle I\rangle$ & $\langle V\rangle$ & Period (days) & Note \\
\hline 1 & AzV480;2dFS3047 & O4-7Ve & 18.729292 & -72.36058 & SMC118.1.5302 & 14.24 & 14.41 & 0.637494 & \\
\hline 2 & 2dFS2553 & O6.5IIf & 17.341583 & -73.26150 & SMC111.3.7734 & 15.01 & 15.02 & 0.823163 & \\
\hline 3 & 2dFS3357 & O9.5III-V & 19.725167 & -73.16056 & SMC121.5.11 & 14.27 & 14.38 & 0.812373 & \\
\hline 4 & J005517.9-723853 & O9.5Ve XRB & 13.824583 & -72.64806 & SMC105.6.33072 & 15.71 & 16.02 & 0.650438 & \\
\hline 5 & AzV322 & O9II & 15.750042 & -72.42761 & SMC113.8.6165 & 13.71 & 13.80 & 2.586981 & \\
\hline 6 & AzV162 & O9V & 13.753292 & -72.93822 & SMC105.8.34986 & 13.53 & 13.75 & 1.598756 & W04 \\
\hline 7 & J0050.7-7316 & B0-0.5 Ve XRB & 12.686250 & -73.26806 & SMC100.2.114 & 15.22 & 15.39 & 0.708424 & W04 \\
\hline 8 & 2dFS1498 & B0-5II & 15.177833 & -72.41572 & SMC108.1.21491 & 15.23 & 15.45 & 0.797337 & I04 \\
\hline 9 & $2 \mathrm{dFS} 3448$ & B0-5II & 20.075375 & -72.31481 & SMC123.1.10 & 14.89 & 14.96 & 1.082169 & \\
\hline 10 & 2dFS3395 & B0-5IIIe & 19.869625 & -73.41081 & SMC121.7.101 & 15.90 & 16.02 & 0.363367 & \\
\hline
\end{tabular}

Notes. Full Table 6 is available at the CDS. A portion is shown here for guidance regarding its form and content.

References. (W04) Wyrzykowski et al. (2004); (D08) Diago et al. (2008); (I04) Ita et al. (2004).

Mennickent et al. (2002), finding 76\% of our irregular variables to be newly discovered variables. Furthermore, $23 \mathrm{Be} / \mathrm{X}$-ray binaries compiled by Raguzova \& Popov (2005) have been identified as having irregular variability, four of which present fading events, and one is a bumper variable in the OGLE-III time domain. Of these 23 systems, 20 have been previously studied photometrically. The OGLE-III light curves of the remaining three systems are displayed for the first time in Fig. 10.

We have also flagged $21 \mathrm{O}, \mathrm{B}$, and A-type supergiants as irregular since their dispersion places them above the variability threshold on the $\sigma_{I}$ vs. I diagram. Through visual inspection, we conclude that for many of these stars, this is just photometric 
Table 7. Long-period stars in the SMC.

\begin{tabular}{|c|c|c|c|c|c|c|c|c|c|}
\hline \# & Star name & Spectral type & RA (J2000) & Dec (J2000) & OGLE-III ID & $\langle I\rangle$ & $\langle V\rangle$ & Period (days) & Note \\
\hline 34 & AzV141;2dFS0915 & B5II & 13.289958 & -72.56311 & SMC101.2.48081 & 14.40 & 14.47 & 31.773741 & I04 \\
\hline 35 & 2dFS0096 & B5III & 8.693208 & -73.39900 & SMC130.2.209 & 16.91 & 17.03 & 174.907623 & $\mathrm{DPV}(+5.1775914 \mathrm{~d})$ \\
\hline 36 & 2dFS0106 & B8II & 8.762917 & -73.61633 & SMC130.1.87 & 15.28 & 15.43 & 26.101492 & \\
\hline 37 & 2dFS0344 & B9II & 10.234250 & -73.12197 & SMC125.5.9333 & 16.64 & 16.68 & 3.555301 & \\
\hline 38 & AzV241;2dFS1437 & B9Ibe & 15.003292 & -72.92311 & SMC105.1.28748 & 14.18 & 14.41 & 35.308189 & I04, M02 \\
\hline 39 & 2dFS0573 & A0II & 11.427792 & -73.57572 & SMC125.1.14657 & 16.24 & 16.29 & 13.125437 & \\
\hline 40 & 2dFS3113 & AOII & 18.909042 & -72.49914 & SMC115.4.5862 & 16.08 & 16.21 & 15.501976 & \\
\hline 41 & 2dFS2073 & A3Iae & 16.468500 & -72.59606 & SMC110.3.12876 & 16.03 & 16.64 & 28.176533 & \\
\hline 42 & SMC3-12 & A3III & 13.496750 & -72.58581 & SMC101.2.48095 & 14.05 & 14.46 & 72.918435 & I04, M02 \\
\hline 43 & 2dFS2354 & A5II & 16.991542 & -72.54867 & SMC110.4.6944 & 14.65 & 14.96 & 33.677862 & \\
\hline
\end{tabular}

Notes. Full Table 7 is available at the CDS. A portion is shown here for guidance regarding its form and content. DPV: Double-periodic variable.

References. (I04) Ita et al. (2004); (M02) Mennickent et al. (2002).

Table 8. Long-period stars with extra variability in the SMC.

\begin{tabular}{|c|c|c|c|c|c|c|c|c|c|}
\hline \# & Star name & Spectral type & RA (J2000) & Dec (J2000) & OGLE-III ID & $\langle I\rangle$ & $\langle V\rangle$ & Period (days) & Note \\
\hline 1 & 2dFS5106 & O6-9 & 15.578500 & -72.29450 & SMC113.7.6154 & 15.19 & 15.33 & 56.007555 & \\
\hline 2 & 2dFS1772 & O9III-V & 15.874167 & -72.29850 & SMC113.7.11951 & 15.05 & 15.07 & 7.009737 & \\
\hline 3 & J005252.1-721715 & Be XRB & 13.217167 & -72.28769 & SMC101.4.25552 & 16.75 & 16.69 & 45.908521 & \\
\hline 4 & 2dFS2064 & B0IV & 16.443292 & -72.11403 & SMC113.3.21 & 14.77 & 14.95 & 27.821916 & \\
\hline 5 & $2 \mathrm{dFS} 2248$ & B0-5V & 16.775125 & -73.07992 & SMC111.4.9965 & 16.83 & 16.86 & 4.420608 & \\
\hline 6 & 2dFS1558 & $\mathrm{B} 0-5 \mathrm{Ve}$ & 15.329042 & -73.85139 & SMC107.3.9244 & 17.54 & 17.52 & 3.433239 & \\
\hline 7 & SMC3-16 & B0IIIe & 14.552417 & -72.51347 & SMC105.4.19191 & 14.55 & 14.85 & 59.631012 & I04, M02 \\
\hline 8 & NGC330-070;SMC5_077231 & B $0.5 \mathrm{e}$ & 14.259125 & -72.43204 & SMC108.8.26225 & 15.96 & 15.99 & 586.438867 & $\mathrm{I} 04, \mathrm{M} 02$ \\
\hline 9 & 2dFS5020 & B1-3II & 12.127417 & -72.92975 & SMC100.5.14632 & 14.33 & 14.35 & 13.706056 & \\
\hline 10 & 2dFS0690;J0048.5-7302 & B1.5e XRB & 12.142750 & -73.04228 & SMC100.6.45825 & 14.73 & 14.89 & 414.913462 & \\
\hline
\end{tabular}

Notes. Full Table 8 is available at the CDS. A portion is shown here for guidance regarding its form and content.

References. (I04) Ita et al. (2004); (M02) Mennickent et al. (2002).

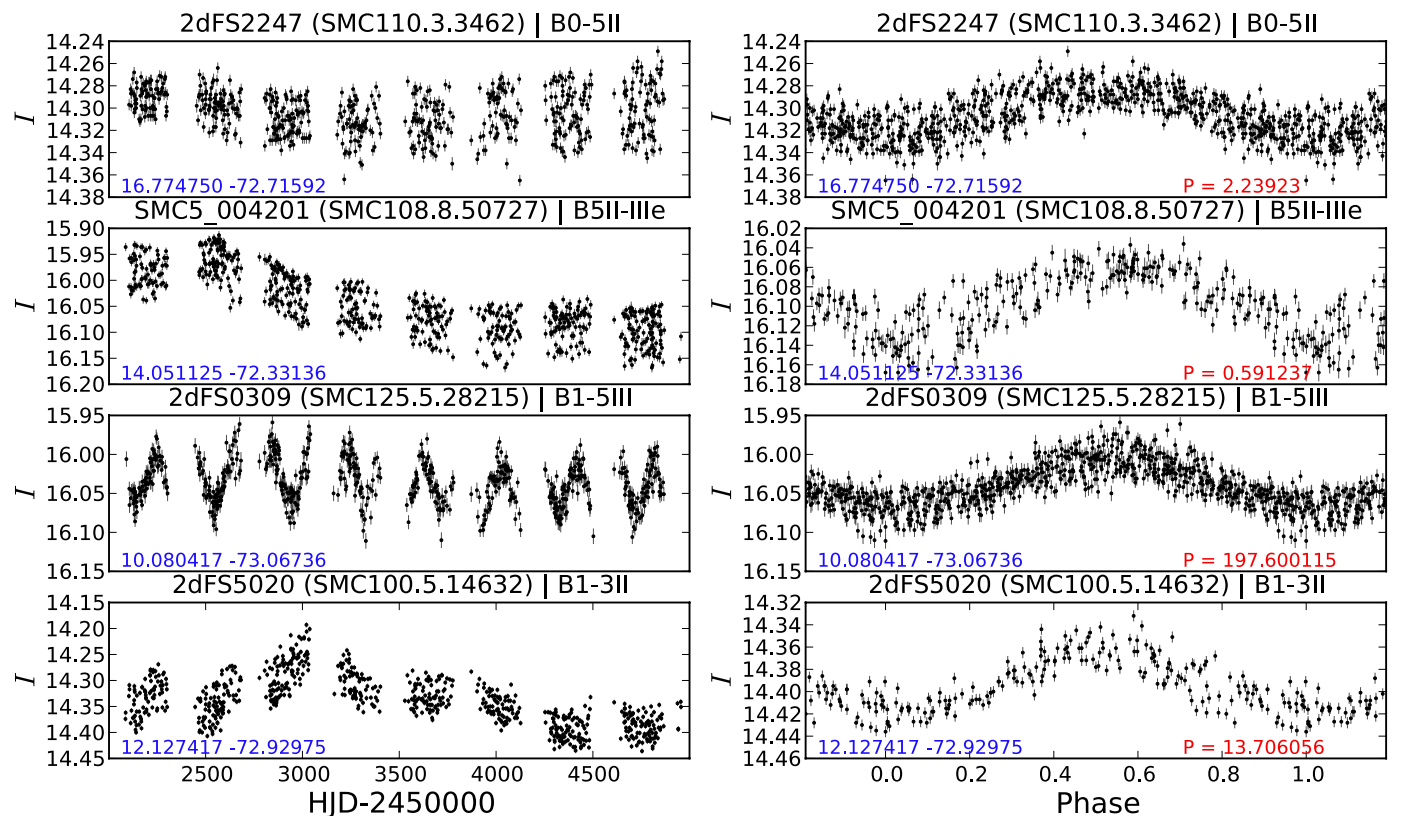

Fig. 8. I-band light curves (left) of 4 periodic variables flagged as "short", "short with extra variability", "long", and "long with extra variability", respectively. These are folded to their derived periods (right). In this sample, the folded light curves of SMC5_004201 and 2dFS5020, which exhibit extra variability, correspond to the HJD ranges 2454 000-2 455000 and $2454200-2455000$, respectively.

noise since they are bright stars close to saturation. However, ten of them do exhibit "Be-like behavior". Furthermore, six of them have been reported as having emission lines in their spectra (although $\mathrm{H} \alpha$ emission could be associated with mass loss, see Leitherer 1988), while one is classified as a B[e] supergiant with a circumstellar envelope (Wisniewski et al. 2007), and its light curve is displayed in Fig. 11. By definition, Be stars are not supergiant stars (Collins 1987), so we caution that 
M. Kourniotis et al.: Variability of massive stars in the SMC
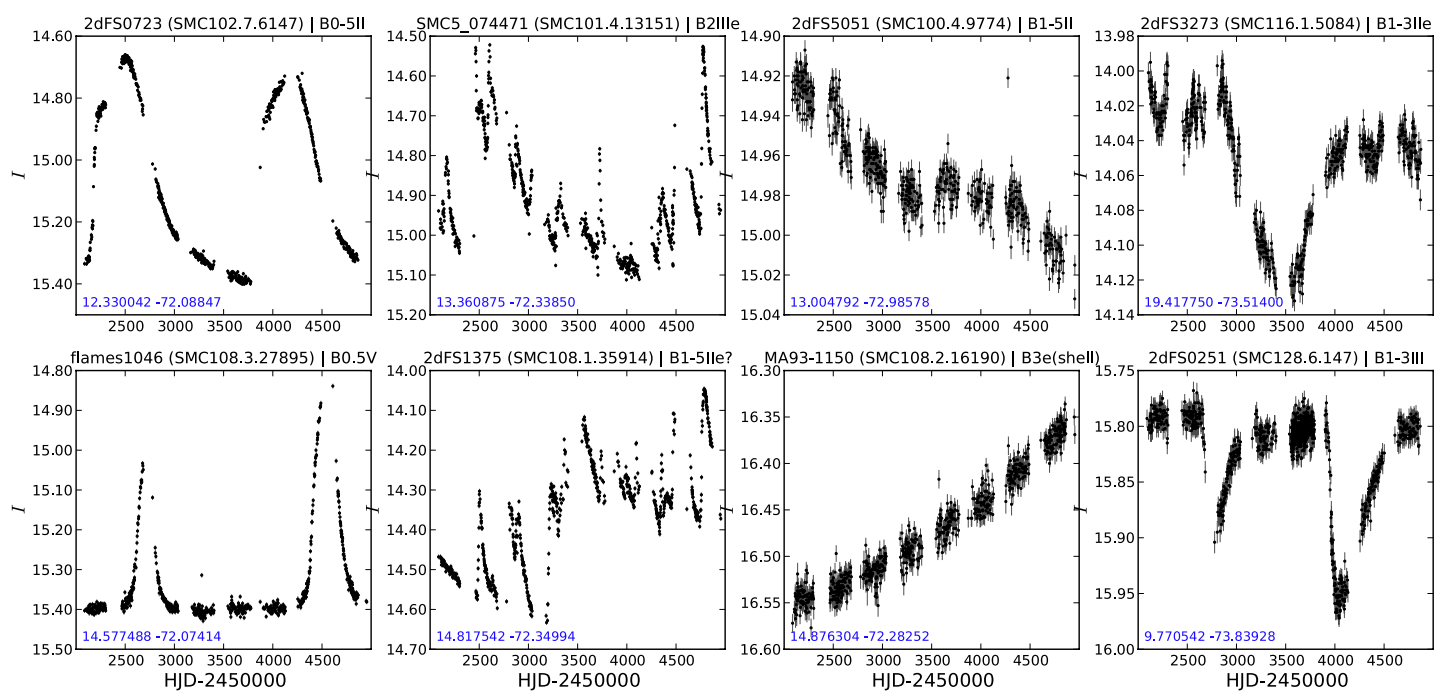

Fig. 9. Representative pairs of light curves of 8 irregularly varying stars presenting (from left to right) bumper, flicker, monotonic, and fading events.
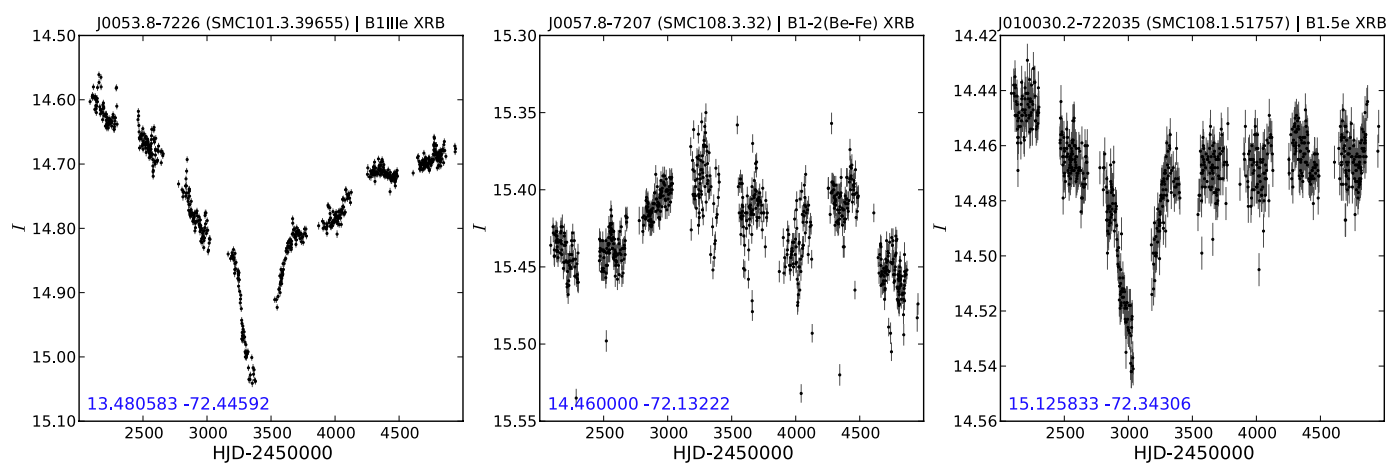

Fig. 10. Light curves of $3 \mathrm{Be} / \mathrm{X}$-ray binaries presented for the first time and classified as irregular variables. The first (left) and third (right) exhibit fading events.
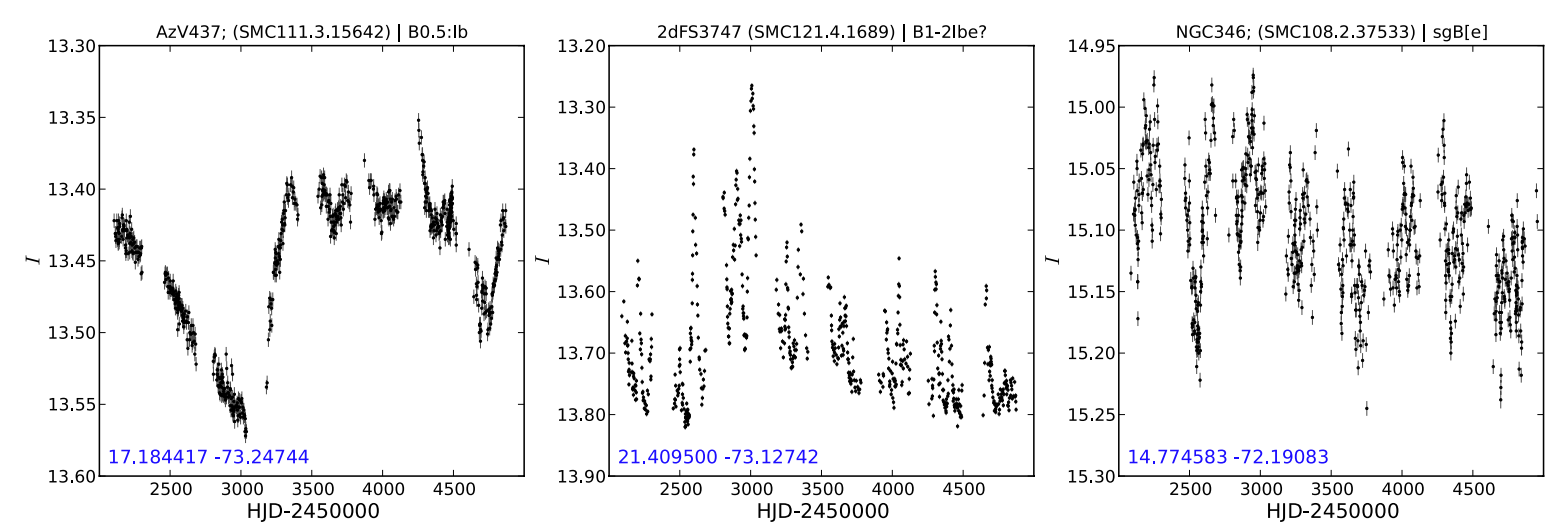

Fig. 11. Light curves of supergiants presenting "Be behavior", including a known sgB[e] star (right panel; Wisniewski et al. 2007). Either the luminosity classification is incorrect or their variability is triggered by mechanisms other than fast rotation, such as magnetic fields along with g-mode non-radial pulsations.

either the luminosity classes of these irregular supergiants may have been overestimated or a mechanism other than fast rotation must be invoked, such as dipole magnetic fields along with non-radial g-mode pulsations (Markova et al. 2008; Saio 2011), which have been reported in the B supergiant region (Saio et al. 2006; Lefever et al. 2007; Moravveji et al. 2012).

Table 10 presents the light curves of all irregular and periodic variables discussed in the previous sections and is available in its entirety at the CDS.

\subsection{Constant stars}

A total of 2766 stars were found below the threshold of variability as defined by the empirical curve in the $\sigma_{I}$ vs. $I$ diagram in Fig. 2. Of these, 109 are O-type stars, 1517 early B-type, 347 late B-type, and 793 are AFG-type stars. In general, their light curves appear constant, although some present evidence for relatively low-amplitude variability. However, this is insufficient to establish them as variables with low amplitude using the criterion of 


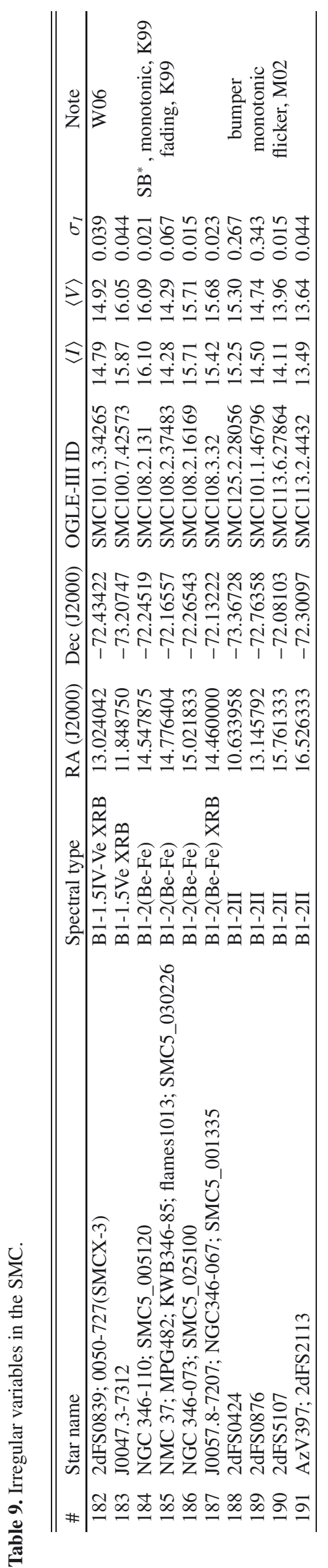

deviation. In the present work, we did not search for periodic signals in the region of constant stars, so we expect that further study will identify pulsating variables with low amplitude $(\Delta I \lesssim$ $0.02 \mathrm{mag}$ ) and eclipsing binaries with short or shallow eclipses among these stars.

\section{Discussion}

Having classified 4646 stars by their variability type, we proceeded to investigate their variability as a function of spectral type. Figure 12 presents four pie charts showing the distribution of the seven types of variability (e.g., "constant", low-amplitude, irregular, short/long-periodic, EBs, and Cepheids) derived through the procedure described in Sect. 4, for 246 O, 2752 early B, 531 late B, and 1108 AFG-type stars. The exact fractions are presented in Table 11. Nine Wolf-Rayet stars do not appear in the statistics, six of which are flagged as lowamplitude variables, two as irregular, and one as a known eclipsing binary. It is striking that variability is so frequent among early-type stars. In fact, since we did not search the "constant" region of the $\sigma_{I}$ vs. $I$ diagram for periodicity, we consider the percentage of constant stars to be an upper limit. Similarly, the fraction of $6.1 \pm 0.5 \%$ of early B-type stars that present shortterm periodic variability corresponds to a lower limit. Stochastic, high-amplitude variability, as currently reflected in the fraction of irregular variables, peaks at $\mathrm{O}$ and early B-type stars, while the decrease beyond the B2 type is considerable. Assuming that irregular variability is mainly caused by the Be phenomenon, we expect it to be associated with an infrared excess and reddened optical colors due to free-free emission originating in the circumstellar region. Indeed, most of the irregular variables exhibit reddening, as shown in Fig. 13, which presents a colormagnitude diagram (CMD) labeling the variables classified in this work. The instability strip of the Cepheids is distinctive, although it hosts a few other variables, which were examined and excluded from being Cepheids. Of these, four resemble RV Tauri variables considering the morphology of their light curves, and they obey the typical PL relation for Type II Cepheids. Nonetheless, they have low amplitudes and eventually, two of these were classified as long-period variables, one as a candidate rotating variable, and one as an eclipsing binary. The "constant" stars and the low-amplitude variables populate the background of the CMD and comprise $\sim 77 \%$ of our studied stars.

We propose irregular and low-amplitude variability for OB-type stars to be a criterion for defining a candidate photometric Be star. If we apply this criterion to our catalog, then the fraction of candidate early Be stars, i.e. early-type stars with irregular or low-amplitude variability, is found to be $30 \pm 1 \%$. This agrees with the fraction $26 \pm 4 \%$ of Be stars obtained in the spectroscopic study of B-type stars by Martayan et al. (2007) in the surrounding field of the open cluster NGC 330 and with the fraction $27 \pm 2 \%$ of Be stars derived by Bonanos et al. (2010) using mid-infrared photometry of early B-type stars. This result is also consistent with the study of four young SMC clusters by Wisniewski \& Bjorkman (2006) with $\mathrm{H} \alpha$ photometry that yielded a fraction $32 \pm 5 \%$ of candidate Be among early B-type stars. We caution that our fraction of Be stars would increase if we included short-periodic stars such as Be pulsating stars, but would also decrease because some irregular and variables with low amplitude may not vary due to the Be mechanism. On the other hand, O-type stars exhibit strong stellar winds driven by radiation pressure that are likely to present low-amplitude variability. After locating our 71 O-type low-amplitude variables on the CMD diagram, only six show evidence of infrared excess. 
Table 10. Lightcurves of variables.

\begin{tabular}{lcccc}
\hline \hline OGLE-III ID & Filter & HJD-2 450 000 (days) & Magnitude (mag) & Uncertainty (mag) \\
\hline SMC100.1.15162 & I & 2085.90914 & 15.081 & 0.006 \\
SMC100.1.15162 & I & 2086.88843 & 15.076 & 0.005 \\
SMC100.1.15162 & I & 2103.89525 & 15.219 & 0.005 \\
SMC100.1.15162 & I & 2105.92315 & 15.052 & 0.005 \\
SMC100.1.15162 & I & 2106.89098 & 15.118 & 0.005 \\
SMC100.1.15162 & I & 2112.84336 & 15.212 & 0.005 \\
SMC100.1.15162 & I & 2116.87111 & 15.206 & 0.005 \\
SMC100.1.15162 & I & 2123.81803 & 15.234 & 0.005 \\
SMC100.1.15162 & I & 2128.82553 & 15.171 & 0.005 \\
SMC100.1.15162 & I & 2129.85750 & 15.048 & 0.005 \\
\hline
\end{tabular}

Notes. Full Table 10 is available at the CDS. A portion is shown here for guidance regarding its form and content.

Table 11. Distribution of variability.

\begin{tabular}{lcccc|cc}
\hline \hline Type of variability & O-type & Early B & Late B & AFG-type & Early Be & Late Be \\
\hline Constant & $44.3 \%$ & $55.1 \%$ & $65.3 \%$ & $71.6 \%$ & $18 \%$ & $33.8 \%$ \\
Low-amplitude & $28.9 \%$ & $16 \%$ & $23 \%$ & $15.2 \%$ & $14.8 \%$ & $24.2 \%$ \\
Irregular & $11.4 \%$ & $14.1 \%$ & $3.8 \%$ & $0.5 \%$ & $45.2 \%$ & $22.6 \%$ \\
Short-periodic & $4.1 \%$ & $6.1 \%$ & $2.1 \%$ & - & $18 \%$ & $11.3 \%$ \\
Long-periodic & $1.6 \%$ & $1.4 \%$ & $1.7 \%$ & $0.8 \%$ & $2.7 \%$ & $3.2 \%$ \\
Rotating cand. & $0.4 \%$ & $1.4 \%$ & $1.5 \%$ & $0.2 \%$ & - & $3.2 \%$ \\
Eclipsing binaries & $9.3 \%$ & $5.9 \%$ & $2.6 \%$ & $0.4 \%$ & $1.1 \%$ & $1.6 \%$ \\
Cepheids & - & - & - & $11.4 \%$ & - & - \\
\hline
\end{tabular}

Notes. The first four columns correspond to the fractions of variability type presented in the pie charts of Fig. 12. The fractions in the right two columns correspond to the pie charts of Fig. 14.

O Type (246)

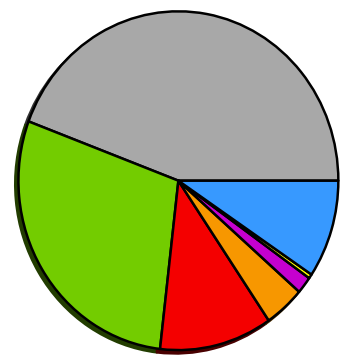

Late B (531)

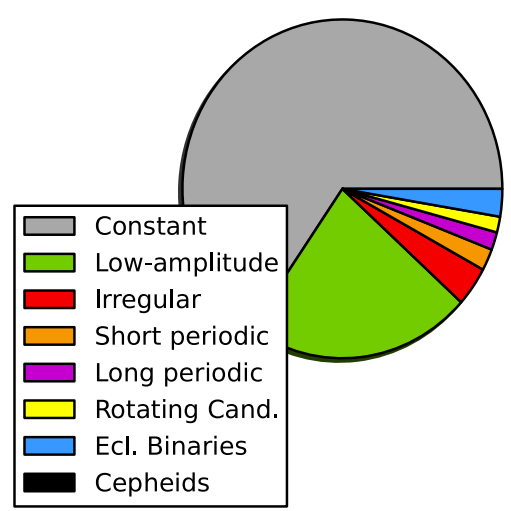

Early B (2752)

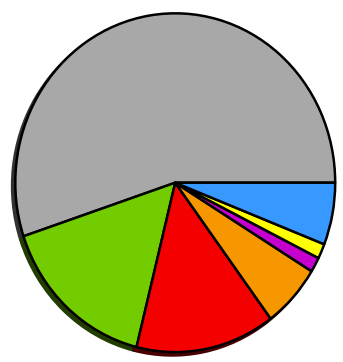

AFG (1108)

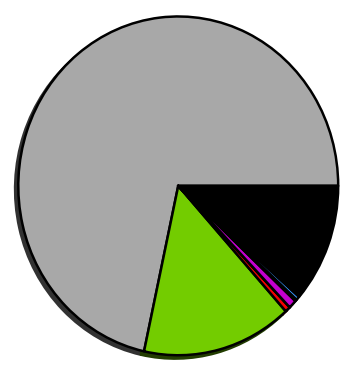

Fig. 12. Pie charts showing the distribution of the type of variability for the $O$, early $B$, late $B$, and AFG-type stars in our catalog. Numbers in parentheses denote the total number of stars for each spectral type. The frequency of variables - particularly the irregular type - increases at earlier spectral types. The exact fractions are presented in Table 11 for clarity. 


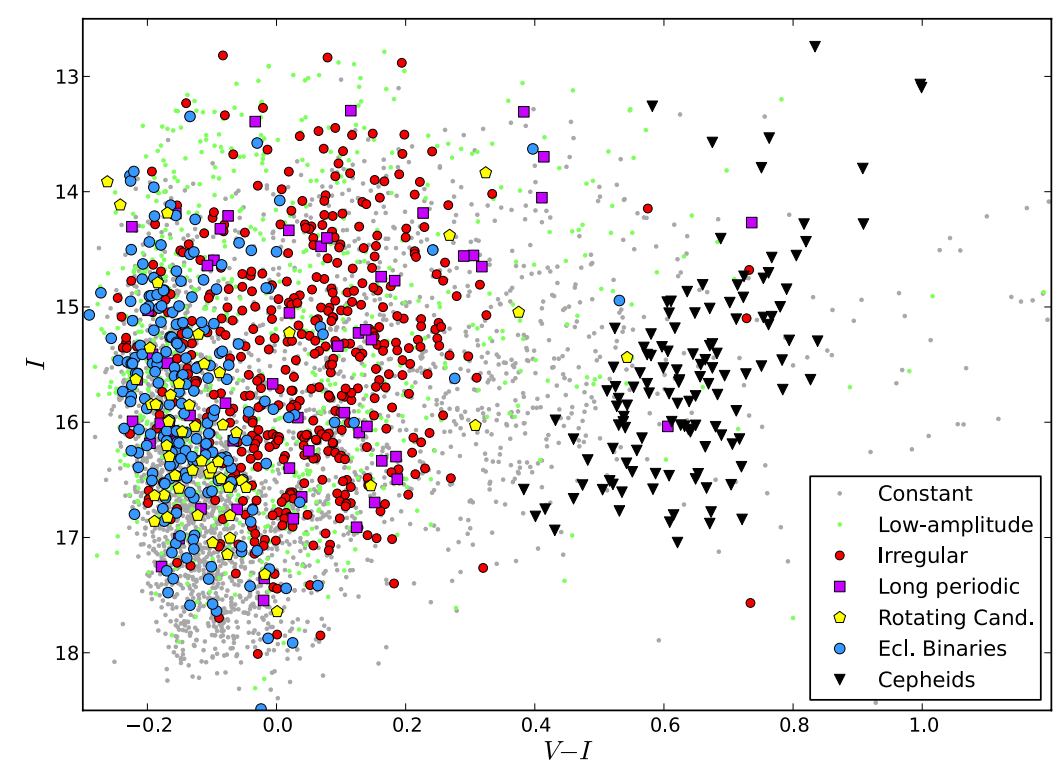

Fig. 13. Color-magnitude diagram as in Fig. 1, but for the type of variability. The variability types labeled are constant stars (gray dots), lowamplitude variables (green dots), irregular variables (red circles), eclipsing binaries (blue circles), Cepheids (black triangles), long-periodic stars $(P>3$ days; purple squares), and candidate rotating variables (yellow pentagons), as defined in Sect. 4. Short-periodic stars do not appear in this diagram for clarity.
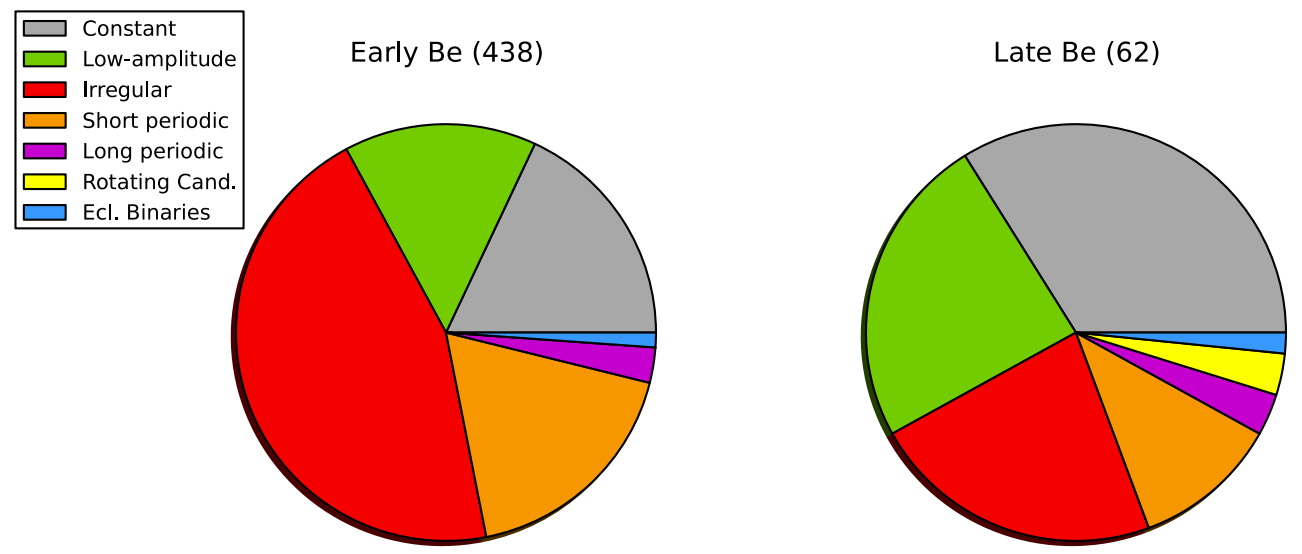

Fig. 14. Pie charts for 438 early B and 62 late B-type stars that are confirmed spectroscopically as Be stars, showing the distribution of variability types across the 8 -year time domain of OGLE-III. We find $78 \%$ of the early Be stars and $58 \%$ of late Be stars to vary photometrically within the photometric precision of OGLE-III, as a result of disk modulation and/or short-term pulsations. The exact fractions are presented in Table 11 for clarity.

Moreover, out of our ten spectroscopically identified Oe stars, only one is flagged as a low-amplitude variable. We therefore do not find low-amplitude variability to be a signature of the Oe phenomenon. We considered our 28 irregular O-type variables, excluding two that are probably close to saturation and adding the six "redder" variables with low amplitude, and found the fraction of candidate Oe stars to be $13 \pm 2 \%$, which is consistent with the fraction $10 \pm 2 \%$ obtained in the mid-infrared study of Bonanos et al. (2010). The agreement of these different methods provides evidence of the validity of the proposed method based on photometric variability.

Figure 14 presents pie charts showing the photometric behavior for the spectroscopically confirmed Be stars with early B and late B types. The exact fractions are presented in Table 11. We find that $45 \pm 3 \%$ of early-Be stars present irregular variability. Adding our stars with stochastic low-amplitude variability, as well as the pulsating Be variables, the fraction of photometrically variable Be stars increases to $78 \pm 4 \%$. In addition, since part of the spectroscopic observations of the Be stars were conducted before the OGLE-III monitoring period, we recalculated the statistics using only the 128 early-Be stars from Evans et al. (2006) and Martayan et al. (2007) that conducted spectroscopy during the OGLE-III monitoring. Such a selection yields a consistent fraction of $79 \pm 8 \%$. On the other hand, the backbone of the catalog comes from Evans et al. (2004) and corresponds to $78 \%$ of the total sample. The spectra were obtained in 1998 and 1999, yielding 228 of our 438 studied earlyBe stars. Using only the Be stars from this survey, the fraction of Be stars that exhibit photometric variability from the subsequent OGLE-III light curves is $79 \pm 6 \%$. We caution, though, that our pulsating fraction is a lower limit since it is incomplete and that the fraction of low-amplitude variable Be stars could be higher, considering that most of them exhibit long-lasting invariant intervals; therefore, when conducting spectroscopy, they have a lower chance of being detected with $\mathrm{H} \alpha$ emission than irregular variables. In addition, Evans et al. (2004) denoted 46 early B-type stars of our sample to have an uncertain ("e?") spectral classification. Of these, one third were classified in our study 

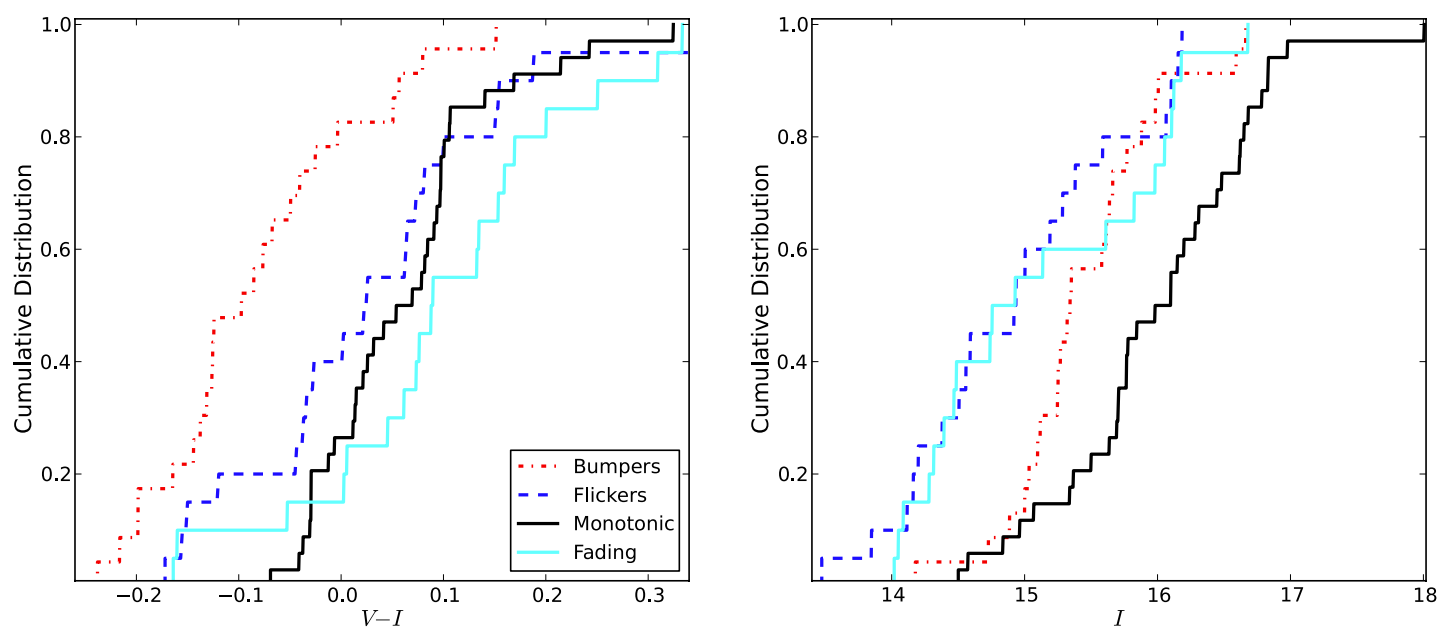

Fig. 15. Cumulative distribution diagrams of stars that present a single mode of irregular variability, as a function of their $V-I$ color (left panel) and their I-band magnitude (right panel). Bumper stars are shown with a dash-dotted red line, flicker types with a dashed blue line, monotonic types with a solid black line, and fading types with a solid cyan line. We note that all four modes exhibit colors between -0.2 and 0.3 mag. The colors of bumper stars are shifted towards the blue only because of their insufficient $V$-band monitoring. Flicker-type stars appear to be the brightest among the modes. About half of the single-mode irregular variables are spectroscopically confirmed as Be stars.

as "constant", while the respective fraction of "constant" stars among the typical "e" stars is one sixth. Therefore, stars with controversial emission, e.g. nebular emission, might have contaminated our Be sample and appear as "constant", although we caution that some of these could indeed vary relatively insignificantly. We therefore report a minimum fraction $\sim 80 \%$ of early B-type stars displaying the Be phenomenon that present significant photometric variability on a eight-year timescale. This percentage is comparable to $86 \%$ provided by Hubert \& Floquet (1998) for short-term variability of Be stars in the Galaxy. The fraction of their short-term period variables in the B0-B3 interval is $\sim 40 \%$, much higher than our incomplete $18 \%$, although the expected fraction $\sim 25 \%$ for pulsating Be stars in the SMC (Diago et al. 2008) is still lower than for the Galaxy, indicating the dependence of the pulsations on metallicity (Pamyatnykh 1999).

It is remarkable that $\sim 53 \%$ of our candidate photometric early Be stars, i.e. low-amplitude and irregular early B-types, are stars of the luminosity class range I-III. As already mentioned, winds and saturation noise are possibly the cause of the lowamplitude variability that is prominent in some of our brightest stars. However, we caution that the presence of a circumstellar envelope causes the Be stars to appear overluminous, while rapid rotation could modify their fundamental parameters by shifting the effective temperature or by leading to an underestimation of vsin $i$ due to the gravitational darkening effect (Townsend et al. 2004). As a result, a fast-rotating star could appear cooler and more luminous. Martayan et al. (2007) implemented fast rotation treatment for their spectroscopic observations in the region of NGC 330, which decreased the fraction of giant Be stars from $\sim 50 \%$ to $\sim 10 \%$. The main sequence Be fraction increased from $\sim 15 \%$ to $\sim 40 \%$, while the fraction of subgiant field Be stars remained at $\sim 45 \%$.

All four modes of irregular variability exhibit a range of $V-I$ colors between -0.2 and 0.3 mag as shown in the cumulative distribution diagram for the average $V-I$ color of the light curves in the left panel of Fig. 15 indicating a range of reddening, which is probably due to the size of the circumstellar disk and its lifetime. The mean color for our bumper type stars is shifted towards the blue because a significant part of their
$V$-band photometry was conducted at intervals when they behaved as regular B stars, i.e. before or after the outbursts, and thus, their color was mainly photospheric. Stars with monotonic behavior seem to be fainter than those exhibiting outbursts, as shown in the cumulative distribution diagram for I magnitudes in the right panel of Fig. 15, while flicker type stars appear brighter than the other modes. Of our single-mode irregular variables, $\sim 53 \%$ are spectroscopically confirmed Be stars.

We identified 11 single-variability mode stars from our catalog as among the Be stars for which Martayan et al. (2007) measured parameters: one bumper, three flicker, five monotonic, and two fading types. The bumper variable SMC5_078440 corresponds to a fast rotator with corrected velocity $v \sin i_{\text {true }} \sim$ $380 \pm 20 \mathrm{~km} \mathrm{~s}^{-1}$. Furthermore, for the three flicker-type stars ${ }^{3}$, the mean obtained velocity is $v \sin i_{\text {true }} \sim 220 \pm 10 \mathrm{~km} \mathrm{~s}^{-1}$, and for the five stars with monotonic behavior ${ }^{4}$ the respective value is $v \sin i_{\text {true }} \sim 290 \pm 10 \mathrm{~km} \mathrm{~s}^{-1}$. Finally, the two fading type variables SMC5_046388 and SMC5_065055 exhibit a true mean velocity $v \sin i_{\text {true }} \sim 400 \pm 30 \mathrm{~km} \mathrm{~s}^{-1}$. Stars with flicker type outbursts seem to be average rotators, and therefore, their parameters are less affected by the rotation. As a result, we suggest that their luminosity is intrinsic rather than due to fast rotation. We suspect that the flickering behavior may occur mostly in evolved stars, although we need a large dataset to confirm this.

de Wit et al. (2006) have proposed a model for outbursts describing the establishment and dissipation of the disk and subsequent modifications of the optical depth. These authors predict that the outflowing disk material produces a clockwise, loop-like structure (vs. counter clockwise loop for the rare case of inflowing material) in the color-magnitude diagram. This bi-valued relation between color and magnitude is produced by the transition of the disk from an optically thick to thin state. Of our 23 bumper variables, we find five stars exhibiting a full-loop, clockwise structure (Fig. 16) with mean color amplitude $\Delta(V-I) \sim$ 0.35 mag and 12 stars presenting very narrow loops or half-loop evidence due to limited $V$-band photometry. The remaining six bumper variables were monitored in the $V$-band mostly before

\footnotetext{
SMC5_074471, SMC5_021152 and SMC5_073581.

4 SMC5_078338, SMC5_075360, SMC5_037137, SMC5_003919 and SMC5_064745.
} 

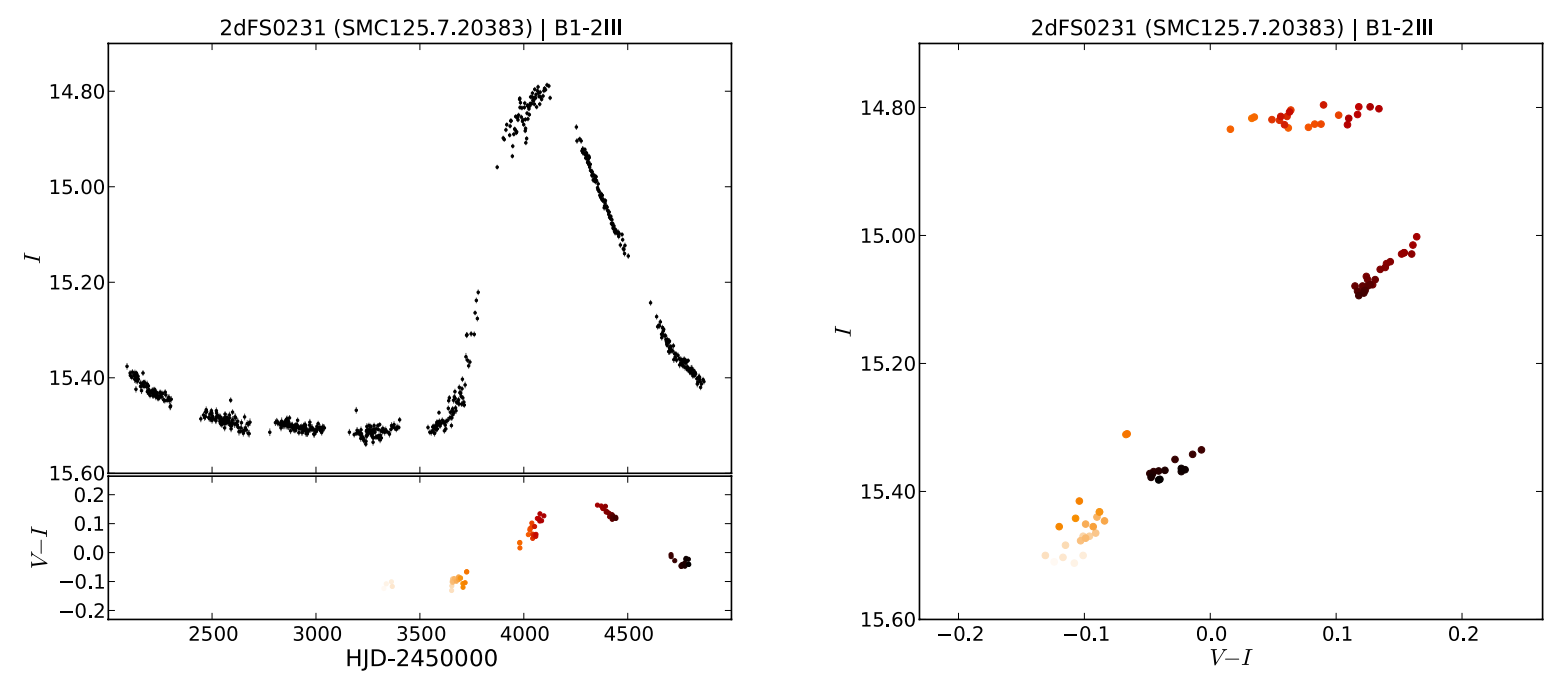

Fig. 16. The left panel shows the $I$-band light curve (upper) and $V-I$ color modulation (lower) of a bumper event exhibiting clockwise loop structure in the color-magnitude diagram, indicating outflowing material that accumulates in a disk that transits from an optically thick to optically thin state (de Wit et al. 2006). The right panel shows the color-magnitude diagram where the loop structure is demonstrated.

or after the outburst. On the other hand, a single-valued relation is most prominent in our monotonic type stars, denoting that ejected matter stays optically thin. Flicker type outbursts have a short duration, and because our $V$-band photometry is sparse, we do not have a clear picture of the CMD pattern or the underlying mechanism. Nonetheless, we did detect clockwise cyclic loops in the flicker-type stars AzV436/2dFS2413 and SMC5_074471 that have spectral types B0IIe and B2IIIe, respectively.

\subsection{Short-periodic stars}

The reason for selecting the limit of three days to distinguish short-period from long-period variables was to differentiate a class of radial pulsating B-type variables with $P<3$ days, known as slowly pulsating B stars (De Cat 2002). Furthermore, non-radial Be pulsators present a common range of periods with SPBs and share the same region in the HR diagram. SPBs are expected to be rare at the low metallicity of the SMC according to computations presented in Miglio et al. (2007). We therefore expect that the discovery of new candidate SPBs could redefine the borders of their instability domain on the HR diagram and update the properties of the metal opacity bump (Salmon et al. 2012). In Fig. 17, we present a CMD comparing the SPBs and the Be pulsators reported by Diago et al. (2008) in the SMC that are also found in our catalog among the short-periodic stars. Of the 188 short-periodic stars, 10 are O-type, 167 are early B-type, and 11 are late B-type stars. Several short-period non-Be (or not defined) stars are colocated with known SPBs and, thus, are candidate SPBs. Similarly, many short-period Be stars are likely to exhibit non-radial pulsations. We also note that the short-periodic stars that show additional variability are redder and consistent with the reported Be pulsators, independent of whether they are found to have Balmer emission. In addition, four Be stars with additional variability studied by Martayan et al. (2007) exhibit mean corrected projected velocities $v \sin i_{\text {true }}=490 \pm 30 \mathrm{~km} \mathrm{~s}^{-1}$, while for 15 short-periodic Be pulsators without additional variability, the respective value is $v \sin i_{\text {true }}=350 \pm 10 \mathrm{~km} \mathrm{~s}^{-1}$. Therefore, we suggest that such evident fluctuations in the light curves are linked to the Be phenomenon and enhanced by fast rotation. Their g-mode non-radial pulsations could be excited by the subsurface iron convection zone, although it is expected to be less prominent at the metallicity of the SMC (Cantiello et al. 2009).

The $\beta$ Cephei stars are another group of blue, short-period, pulsating variables. These are not expected at the metallicity of the SMC; however, several candidate $\beta$ Cephei stars have been reported in the SMC (Kołaczkowski et al. 2006; Sarro et al. 2009; Diago et al. 2008), and their presence possibly denotes metal-rich stars in the galaxy but could also denote an uncertainty in the opacity of nickel by a factor of two (Salmon et al. 2012). Among our short-periodic stars, we have identified seven candidate $\beta$ Cephei stars. These are early-type, multiperiodic variables with blue colors $(V-I<0)$ that present low-amplitude sinusoidal periodicity up to 0.5 days. We flag these in Table 5 and provide the two periods that correspond to the highest frequency peaks.

\subsection{Eclipsing binaries}

Through the present work, we doubled the number of the massive EBs known in the SMC from 104 to 205. The vast majority of them lie close to the main sequence. Figure 18 presents their period-magnitude relation as a function of their spectral type. The majority of O-type binaries are short-period systems, while B-type systems reach an upper limit of about 130 days. On the other hand, the four $\mathrm{A}$ and F-type eclipsing binaries identified have long periods. Two of these, 2dFS0142 and SMC3-4 (Mennickent et al. 2010), are supergiants with wide orbits, and one contains composite features of $\mathrm{A}$ and F-type stars, as discussed in Sect. 4.1.1. The newly discovered F0 type EB 2dFS2348, along with the likely ellipsoidal variable 2dFS0951 of type A7Ib reported by Wyrzykowski et al. (2004) exhibit similar colors $V-I \sim 0.5$. The morphology of their light curves reveals they are contact binaries, while the unequal eclipse depths suggest systems with one evolved component overflowing its Roche lobe along with a bluer companion, which shifts the mean color. We proceeded to compare these to the yellow SMC supergiant R47 (Prieto et al. 2008) of similar color, with absolute magnitude $M_{V}=-7.5$, which is identified as a likely progenitor of Type IIP supernova. Nonetheless, our systems have rather low amplitude, and their $V$-band magnitudes indicate less massive components with $M_{V} \sim-3.5$. 
M. Kourniotis et al.: Variability of massive stars in the SMC

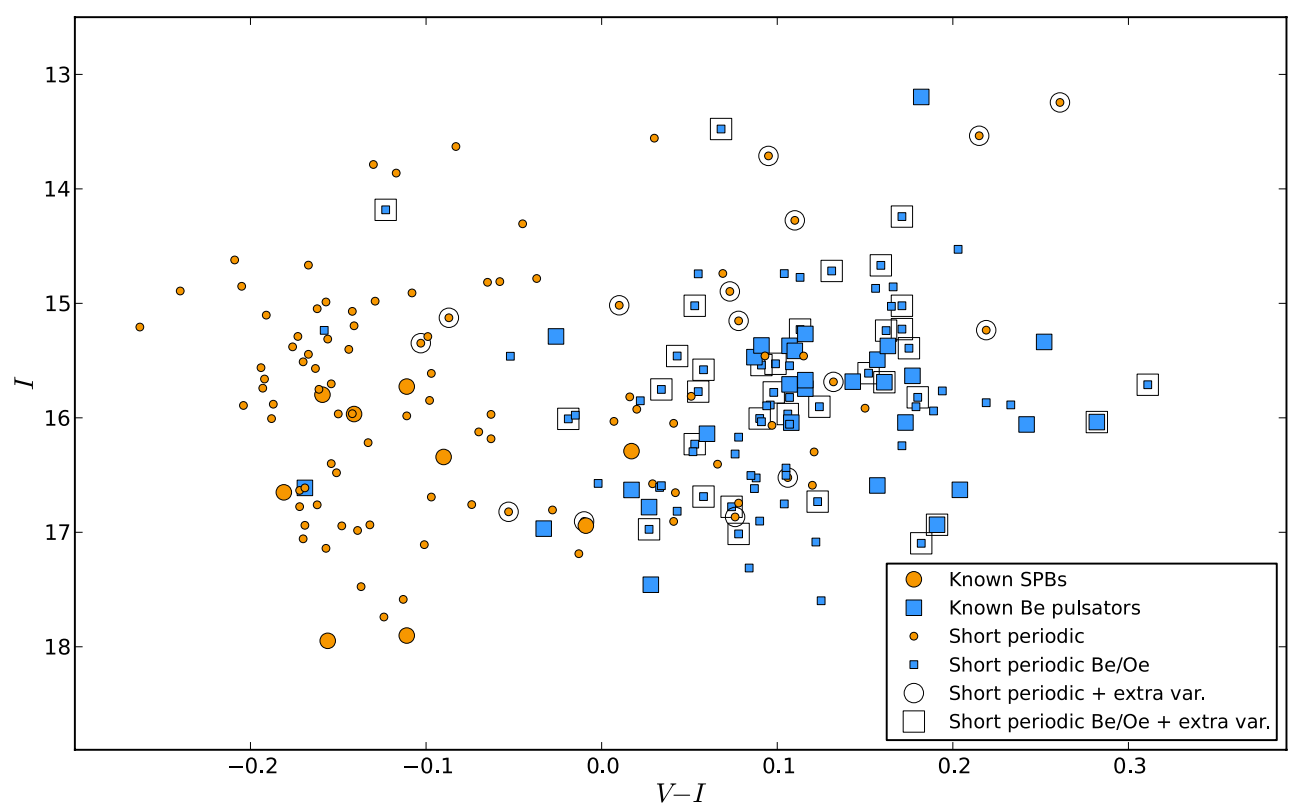

Fig. 17. Color-magnitude diagram for the short-periodic stars $(P<3$ days $)$ in this paper along with the known SPBs and non-radial Be pulsating stars from Diago et al. (2008). Several early B-type stars are likely to be newly discovered SPBs. Also, short-periodic stars with additional variability exhibit redder colors, indicating the origin of such behavior to the Be phenomenon.

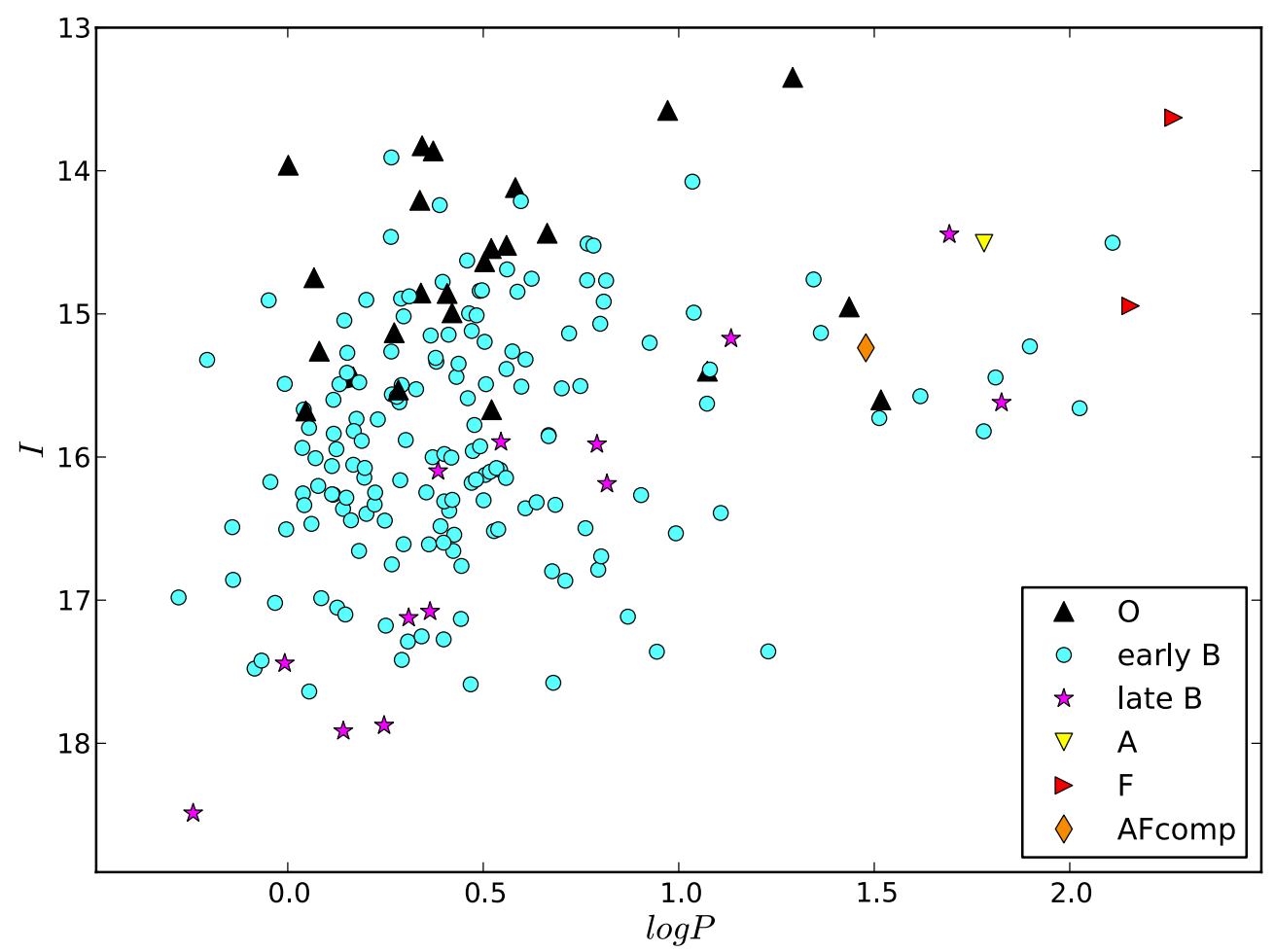

Fig. 18. Period-magnitude diagram for the known and the newly discovered eclipsing binaries of our sample, showing a prominent concentration of early type EBs in short-period orbits and a distinctive tail of luminous EBs towards longer periods. All four AFG-type EBs exhibit periods over $\sim 30$ days.

Short-period binaries with closer orbits containing large, massive stars, as in the case of O-type systems, are more likely to be detected in eclipsing configurations. They therefore dominate our eclipsing sample. To evaluate the fraction of eclipsing binaries in each spectral bin, we eliminated possible selection effects of the compiled input catalog by removing the eclipsing binaries studied by Hilditch et al. (2005) and Harries et al. (2003), as well as additional selected $\mathrm{X}$-ray binaries and periodic variables. The observed fractions of eclipsing systems are $5.2 \pm 1.5 \%$ for O-type, $4.8 \pm 0.4 \%$ for early B-type, $2.7 \pm 0.7 \%$ for late B-type, and unsurprisingly, only $0.3 \pm 0.2 \%$ for AFG-type stars since evolved and luminous stars mainly belong to wide, longperiod systems (Wyrzykowski et al. 2004; Udalski et al. 1998) and, therefore, are rarely identified. 


\section{Conclusions}

We used the photometry provided by the OGLE-III monitoring survey to study the variability of 4646 massive stars with known spectral types in the SMC. This study of the variability of massive stars with known spectral types is a factor of 7 larger than the work of Szczygieł et al. (2010). A total of 2766 stars that correspond to $\sim 60 \%$ of our sample present invariant light curves given the accuracy of the photometry. We detected stochastic low-amplitude variability with a mean $\Delta I \lesssim 0.05$ in 807 stars and variability of higher amplitude in 443 stars. We also report the discovery of $101 \mathrm{EBs}^{5}$ with massive components and present the OGLE-III photometry for 104 known EBs. We hope these will motivate follow-up studies to determine their fundamental parameters and contribute to the small sample of massive stars with accurately measured parameters. Moreover, we have detected short-term periodicity ( $P<3$ days) in 188 stars, thereby motivating studies on new candidate SPBs and non-radially pulsating Be variables. Having inspected four pulsating Be stars with fluctuating amplitude or baseline, we find that they are rapid rotators close to their critical speed, suggesting that fast rotation is responsible for their additional variability most likely caused by matter ejections.

Long-term photometry is established as a powerful tool for studying massive stars, determining accurate periods for known $\mathrm{EBs}$, and discovering rare long-period systems. It provides a new method for discovering new candidate Be stars that were not detected before, either because they were not subject to $\mathrm{H} \alpha$ spectroscopy or they were observed during their transition back to their B state owing to the dissipation of their circumstellar gas disk. A fraction of $51 \pm 4 \%$ of our irregular early B stars have been already identified spectroscopically as Be stars. The remaining irregulars ( $\sim 190$ stars) therefore comprise new candidate Be stars. Furthermore, 20 O-type stars that exhibit irregular variability are likely new candidate Oe stars. On the other hand, the fraction of low-amplitude early B-type variables identified as Be stars is only $15 \pm 2 \%$ mainly because their sparse and/or short-term fluctuations make their spectroscopic identification as Be stars unlikely. We note, though, that not all low-amplitude variables are expected to be linked to the Be phenomenon.

This work is just a foretaste of the results that LSST (Ivezic et al. 2008) and Pan-STARRS (Magnier et al. 2013) will produce for the nearby galaxies that they will survey. Similar follow-up research is possible to be carried out on the LMC, based on the catalog of Bonanos et al. (2009), which consists of massive stars with known spectral type, with the goal of studying and comparing the Be phenomenon from the photometric perspective among environments of different metallicity.

Acknowledgements. We thank the anonymous referee and R. Mennickent for helpful comments that have improved the manuscript. M. Kourniotis and A. Z. Bonanos acknowledge research and travel support from the European Commission Framework Program Seven under the Marie Curie International Reintegration Grant PIRG04-GA-2008-239335. The OGLE project has received funding from the European Research Council under the European Community's Seventh Framework Program (FP7/2007-2013)/ERC grant agreement No. 246678. This research made use of the VizieR catalog access tool at the CDS, Strasbourg, France, and of NASA's Astrophysics Data System Bibliographic Services.

\footnotetext{
5 These EBs became part of the recently released OGLE-III set of EBs in the SMC (Pawlak et al. 2013).
}

\section{References}

Adams, J. J., Simon, J. D., Bolatto, A. D., et al. 2013, ApJ, 771, 112 Alcock, C., Akerlof, C. W., Allsman, R. A., et al. 1993, Nature, 365, 621 Andersen, J. 1991, A\&ARv, 3, 91

Andrievsky, S. M., Kovtyukh, V. V., Luck, R. E., et al. 2002, A\&A, 392, 491

Aubourg, E., Bareyre, P., Brehin, S., et al. 1995, A\&A, 301, 1

Bayne, G., Tobin, W., Pritchard, J. D., et al. 2002, MNRAS, 331, 609

Bjorkman, J. E., \& Cassinelli, J. P. 1993, ApJ, 409, 429

Bonanos, A. Z. 2009, ApJ, 691, 407

Bonanos, A. Z., Stanek, K. Z., Udalski, A., et al. 2004, ApJ, 611, L33 Bonanos, A. Z., Stanek, K. Z., Kudritzki, R. P., et al. 2006, ApJ, 652, 313

Bonanos, A. Z., Massa, D. L., Sewilo, M., et al. 2009, AJ, 138, 1003

Bonanos, A. Z., Lennon, D. J., Köhlinger, F., et al. 2010, AJ, 140, 416

Bonanos, A. Z., Castro, N., Macri, L. M., \& Kudritzki, R.-P. 2011, ApJ, 729, L9

Cantiello, M., Langer, N., Brott, I., et al. 2009, A\&A, 499, 279

Cassinelli, J. P., Brown, J. C., Maheswaran, M., Miller, N. A., \& Telfer, D. C. 2002, ApJ, 578, 951

Catalano, F. A., Kroll, R., \& Leone, F. 1991, A\&A, 248, 179

Chini, R., Hoffmeister, V. H., Nasseri, A., Stahl, O., \& Zinnecker, H. 2012, MNRAS, 424, 1925

Collins, II, G. W. 1987, in Physics of Be Stars, eds. A. Slettebak, \& T. P. Snow, Proc. IAU Colloq. 92 (Cambridge University Press), 3

De Cat, P. 2002, in IAU Colloq. 185: Radial and Nonradial Pulsationsn as Probes of Stellar Physics, eds. C. Aerts, T. R. Bedding, \& J. Christensen-Dalsgaard, ASP Conf. Ser., 259, 196

de Wit, W. J., Beaulieu, J.-P., Lamers, H. J. G. L. M., Lesquoy, E., \& Marquette, J.-B. 2003, A\&A, 410, 199

de Wit, W. J., Lamers, H. J. G. L. M., Marquette, J. B., \& Beaulieu, J. P. 2006 A\&A, 456, 1027

Diago, P. D., Gutiérrez-Soto, J., Fabregat, J., \& Martayan, C. 2008, A\&A, 480, 179

Evans, C. J., Howarth, I. D., Irwin, M. J., Burnley, A. W., \& Harries, T. J. 2004, MNRAS, 353, 601

Evans, C. J., Lennon, D. J., Smartt, S. J., \& Trundle, C. 2006, A\&A, 456, 623

Faccioli, L., Alcock, C., Cook, K., et al. 2007, AJ, 134, 1963

Graczyk, D., \& Eyer, L. 2010, Acta Astron., 60, 109

Groenewegen, M. A. T. 2005, A\&A, 439, 559

Harries, T. J., Hilditch, R. W., \& Howarth, I. D. 2003, MNRAS, 339, 157

Hilditch, R. W., Howarth, I. D., \& Harries, T. J. 2005, MNRAS, 357, 304

Hubert, A. M., \& Floquet, M. 1998, A\&A, 335, 565

Ita, Y., Tanabé, T., Matsunaga, N., et al. 2004, MNRAS, 353, 705

Ivezic, Z., Tyson, J. A., Acosta, E., et al. 2008 [arXiv: 0805. 2366] available at http://www. lsst.org/overview

Keller, S. C., Wood, P. R., \& Bessell, M. S. 1999, A\&AS, 134, 489

Keller, S. C., Bessell, M. S., Cook, K. H., Geha, M., \& Syphers, D. 2002, AJ, 124, 2039

Kołaczkowski, Z., Pigulski, A., Soszyński, I., et al. 2006, Mem. Soc. Astron. It., 77, 336

Krtička, J., Mikulášek, Z., Henry, G. W., et al. 2009, A\&A, 499, 567

Krtička, J., Mikulášek, Z., Lüftinger, T., et al. 2012, A\&A, 537, A14

Kudritzki, R.-P., \& Puls, J. 2000, ARA\&A, 38, 613

Lefever, K., Puls, J., \& Aerts, C. 2007, A\&A, 463, 1093

Leitherer, C. 1988, ApJ, 326, 356

Lüftinger, T., Fröhlich, H.-E., Weiss, W. W., et al. 2010, A\&A, 509, A43

Magnier, E. A., Schlafly, E., Finkbeiner, D., et al. 2013, ApJS, 205, 20

Markova, N., Prinja, R. K., Markov, H., et al. 2008, A\&A, 487, 211

Martayan, C., Floquet, M., Hubert, A. M., et al. 2007, A\&A, 472, 577

Martayan, C., Baade, D., \& Fabregat, J. 2010, A\&A, 509, A11

McSwain, M. V., \& Gies, D. R. 2005, ApJS, 161, 118

Mennickent, R. E., Pietrzyński, G., Gieren, W., \& Szewczyk, O. 2002, A\&A, 393, 887

Mennickent, R. E., Pietrzyński, G., Diaz, M., \& Gieren, W. 2003, A\&A, 399, L47

Mennickent, R. E., Cidale, L., Pietrzyński, G., Gieren, W., \& Sabogal, B. 2006, A\&A, 457, 949

Mennickent, R. E., Kołaczkowski, Z., Michalska, G., et al. 2008, MNRAS, 389, 1605

Mennickent, R. E., Smith, M. A., Kołaczkowski, Z., Pietrzyński, G., \& Soszyński, I. 2010, PASP, 122, 662

Miglio, A., Montalbán, J., \& Dupret, M.-A. 2007, MNRAS, 375, L21

Moravveji, E., Moya, A., \& Guinan, E. F. 2012, ApJ, 749, 74

Niemela, V. S., Massey, P., Testor, G., \& Giménez Benítez, S. 2002, MNRAS, 333, 347

Pamyatnykh, A. A. 1999, Acta Astron., 49, 119

Pawlak, M., Graczyk, D., Soszyński, I., et al. 2013, Acta Astron., 63, 323

Pojmanski, G. 2002, Acta Astron., 52, 397

Poleski, R., Soszyñski, I., Udalski, A., et al. 2010, Acta Astron., 60, 179 
Porter, J. M., \& Rivinius, T. 2003, PASP, 115, 1153

Prieto, J. L., Stanek, K. Z., Kochanek, C. S., et al. 2008, ApJ, 673, L59

Prša, A., Batalha, N., Slawson, R. W., et al. 2011, AJ, 141, 83

Raguzova, N. V., \& Popov, S. B. 2005, Astron. Astrophys. Trans., 24, 151

Rauw, G., De Becker, M., Nazé, Y., et al. 2004, A\&A, 420, L9

Richardson, N. D., Morrison, N. D., Kryukova, E. E., \& Adelman, S. J. 2011, AJ, 141,17

Rivinius, T., Baade, D., Stefl, S., et al. 1998, A\&A, 333, 125

Rivinius, T., Baade, D., Štefl, S., et al. 2001, A\&A, 369, 1058

Rivinius, T., Carciofi, A. C., \& Martayan, C. 2013, A\&ARv, 21, 69

Romaniello, M., Primas, F., Mottini, M., et al. 2008, A\&A, 488, 731

Sabogal, B. E., Mennickent, R. E., Pietrzyński, G., \& Gieren, W. 2005, MNRAS, 361,1055

Sabogal, B. E., Mennickent, R. E., Pietrzyński, G., et al. 2008, A\&A, 478, 659

Saio, H. 2011, in IAU Symp. 272, eds. C. Neiner, G. Wade, G. Meynet, \& G. Peters, 468

Saio, H., Kuschnig, R., Gautschy, A., et al. 2006, ApJ, 650, 1111

Salmon, S., Montalbán, J., Morel, T., et al. 2012, MNRAS, 422, 3460

Samus, N. N., Durlevich, O. V., et al. 2009, VizieR Online Data Catalog: II/25

Sana, H., de Mink, S. E., de Koter, A., et al. 2012, Science, 337, 444

Sarro, L. M., Debosscher, J., López, M., \& Aerts, C. 2009, A\&A, 494, 739

Schwarzenberg-Czerny, A. 1989, MNRAS, 241, 153
Sheets, H. A., Bolatto, A. D., van Loon, J. T., et al. 2013, ApJ, 771, 111 Shulyak, D., Krtička, J., Mikulášek, Z., et al. 2010, A\&A, 524, A66 Soszynski, I., Udalski, A., Kubiak, M., et al. 2004, Acta Astron., 54, 347 Soszyński, I., Poleski, R., Udalski, A., et al. 2010, Acta Astron., 60, 17 Soszyński, I., Udalski, A., Szymański, M. K., et al. 2011, Acta Astron., 61, 217 Szczygieł, D. M., Stanek, K. Z., Bonanos, A. Z., et al. 2010, AJ, 140, 14 Torres, G., Andersen, J., \& Giménez, A. 2010, A\&ARv, 18, 67 Townsend, R. H. D., Owocki, S. P., \& Howarth, I. D. 2004, MNRAS, 350, 189 Udalski, A. 2003, Acta Astron., 53, 291

Udalski, A., Kubiak, M., \& Szymanski, M. 1997, Acta Astron., 47, 319 Udalski, A., Soszynski, I., Szymanski, M., et al. 1998, Acta Astron., 48, 563

Udalski, A., Soszyński, I., Szymański, M. K., et al. 2008, Acta Astron., 58, 329 Vink, J. S., de Koter, A., \& Lamers, H. J. G. L. M. 2001, A\&A, 369, 574

Watson, C. L. 2006, Proc. Society for Astronomical Sciences Annual Symposium, 25, 47

Wisniewski, J. P., \& Bjorkman, K. S. 2006, ApJ, 652, 458

Wisniewski, J. P., Bjorkman, K. S., Bjorkman, J. E., \& Clampin, M. 2007, ApJ, 670,1331

Wyrzykowski, L., Udalski, A., Kubiak, M., et al. 2004, Acta Astron., 54, 1

Zaritsky, D., Harris, J., Thompson, I. B., Grebel, E. K., \& Massey, P. 2002, AJ, 123,855 